\title{
Voluntary disclosures and information production by analysts 2
}

\author{
Nisan Langberg, K. Sivaramakrishnan* \\ C.T. Bauer College of Business, University of Houston, 334 Melcher Hall, Houston, TX 77204-6021, USA
}

Received 1 February 2006; received in revised form 11 November 2007; accepted 12 November 2007

Available online 25 January 2008

\begin{abstract}
We analyze the voluntary disclosure decision of a manager when analysts scrutinize the quality of disclosure. We derive an equilibrium in which managers voluntarily disclose unfavorable information only if sufficiently precise, but disclose favorable news with lower levels of accuracy. We show that analysts cover good news disclosures with higher scrutiny. To the extent analysts rely on mandatory financial reports to interpret voluntary disclosures, we show that more precise financial reports may lead to more precise but less frequent voluntary disclosures. Moreover, a slant toward conservatism in financial reports can lead to less precise yet more frequent voluntary disclosures.
\end{abstract}

(C) 2007 Elsevier B.V. All rights reserved.

JEL classification: G14

Keywords: Voluntary disclosure; Conservatism; Accounting precision; Financial analysts

\section{Introduction}

The recent wave of corporate finance scandals and accounting restatements has increased the demand for transparency in financial markets. In fact, the Congress recently passed an "act to protect investors by improving the accuracy and reliability of corporate disclosures" (Sarbanes Oxley Act of 2002). These events have inspired research in accounting and finance to focus on the quality and transparency of financial reports. However, and perhaps surprisingly, voluntary disclosures have not come under similar scrutiny. Extant models of voluntary disclosure have advanced our understanding of why some firms disclose private information voluntarily while other firms do not. However, less is known about how outsiders' interpretation of voluntarily disclosed information might affect the quality of these disclosures, and whether regulations and standards governing financial reporting might have a bearing on a firm's propensity to disclose voluntarily.

\footnotetext{
${ }^{2}$ We thank Anil Arya, Ramji Balakrishnan, Jan Bouwens, Martin Dierker, George Drymiotes, John Fellingham, Tom George, Jonathan Glover, Praveen Kumar, Naftali Langberg, Laurence Van Lent, Tong Lu, and workshop participants at the Ohio State University, Tilburg University. We especially thank Ronald Dye (referee) and S.P. Kothari (editor) for many valuable comments and suggestions.

${ }^{*}$ Corresponding author. Tel.: + 17137434630 ; fax: + 17137434828.

E-mail addresses: nlangberg@uh.edu (N. Langberg), shiva@uh.edu (K. Sivaramakrishnan).
} 
These issues have been raised in Verrecchia (2001) and Dye (2001), which offer insightful discussions of extant voluntary disclosure models.

We take a first step in this direction by analyzing voluntary disclosure in a model that incorporates the information acquisition and processing roles of financial analysts. Financial analysts play an important role in shaping market expectations. ${ }^{1}$ They arguably engage in costly information acquisition and processing to issue forecasts and stock recommendations. Clearly, managers would take analysts' intermediary role into account when strategically disclosing information. Traditional models of voluntary disclosure assume that firms can credibly disclose information (when they choose to disclose), and disclosures are thus accurate at all times. ${ }^{2}$ This assumption, however, does not admit a role for financial analysts as there is little to evaluate or analyze once disclosure occurs. In this paper, our purpose is to introduce a role for financial analysts by allowing for some residual information asymmetry even after disclosure.

To achieve this end, we consider two types of managements' private information with respect to firm value - "hard" information and "soft" information. Growth in earnings, market share and business alliances are examples of hard information that often appear in Management Disclosure and Analysis (MDA) section of financial reports. Disclosures in this section are mostly voluntary. Assessing the implications of such disclosures for firm value requires additional knowledge of soft information. We refer to this soft information as the quality of hard information, or simply, quality of information. ${ }^{3}$ A higher level of quality casts hard information in more favorable light (e.g., greater permanency in earnings growth and higher sustainability in market share). Hard information is (credibly) communicable via disclosure while its quality is difficult to communicate in a credible manner.

It is this inability to credibly communicate the quality dimension that creates a potential role for analysts. By engaging in costly information acquisition and analysis, analysts can learn about the quality of disclosed information. The question of interest is whether and how a firm's voluntary disclosure policy is affected by analysts' interpretation of disclosed information via their costly information acquisition role and the consequent implications for prices.

As a first step toward addressing this question, we build on the voluntary disclosure model of Dye (1985) to show the existence of a voluntary disclosure equilibrium in which the firm discloses hard information only when its quality is above a certain quality threshold. An analysis of this equilibrium yields some immediate insights. First, voluntary disclosures are inherently conservative in this equilibrium, i.e. disclosure of information with poor (unobservable) quality is less likely.

Second, equilibrium quality thresholds are lower for high realizations of hard news (hereafter, good news), but are more conservative for low realizations of hard news (hereafter, bad news). In other words, relative to good news disclosures, bad news disclosures are more precise because of less uncertainty regarding the quality dimension. Also, bad news disclosures are more informative because the disclosed hard information is more indicative of firm value. And, firms will disclose bad news less frequently. There is direct evidence in support for these implications from the empirical literature on voluntary disclosures. In particular, Kothari et al. (2005) examine a sample of (voluntary) management forecasts and show that firms tend to withhold bad news forecasts relative to good news forecasts and also that bad news forecasts are relatively more accurate.

The above equilibrium properties point to a natural demand for analyst scrutiny. Since the analyst's information acquisition effort pertains to the quality dimension, we would expect the demand for scrutiny to be more pronounced when a firm makes a good news disclosure. This in fact is the case in equilibrium. In particular, as the disclosure becomes less informative for higher realizations of the hard information, the analyst's optimal level of scrutiny increases as an equilibrium response. An appealing aspect of this result is that it points to the tendency of managers, acting in the interests of current shareholders, to make relatively less precise good news disclosures, that, in turn, calls for a greater level of scrutiny from the analyst community. It also provides a rationale for the observed emphasis on conservatism in accounting and audit

\footnotetext{
${ }^{1}$ Numerous studies in accounting and finance employ analysts' earnings forecasts as a proxy for market's expectations. Indeed, recent studies suggest that managers care tremendously about meeting or exceeding analysts' expectations (Bartov et al., 2002; Lopez and Rees, 2002).

${ }^{2}$ Verrecchia (2001) highlights this assumption as one of the three main features of extant voluntary or discretionary disclosure models.

${ }^{3}$ For expositional convenience, we sometimes refer to "hard" news as simply "news." However, we will make the reference explicit wherever required to avoid any confusion.
} 
policies. That is, if allowing managers discretion in reporting were to result in more imprecise good news disclosures, then one can argue that mandating a conservative bias that requires an understatement of good news can serve to curb such behavior. ${ }^{4}$ This is in line with Basu (1997) who interprets stronger market responses to positive earnings surprises relative to negative earnings surprises as evidence of conservatism in mandated financial accounting reports.

An important source of information for analysts in their information gathering endeavor is periodical financial reports of firms. It is reasonable to expect that the nature of accounting standards and rules that govern these reports are likely to influence their assessments. For example, mandating more detailed financial reports is likely to enhance analysts' ability to interpret voluntary disclosures by firms. Reporting biases such as accounting conservatism (Watts, 2003a) will also likely have an effect. We explore this intuitive link between the voluntary disclosure regime and accounting systems (via the analyst's information acquisition and processing role) to address some hitherto unanswered questions ${ }^{5}$ : Do strict disclosure requirements lead to more accurate and more frequent voluntary disclosures? Does conservatism in audited financial reports lead to biases in voluntary disclosures? Specifically, we extend our basic model to examine how precision and bias in financial reporting systems might influence analyst scrutiny of voluntary disclosures (i.e., analysts' ability to assess quality of voluntary disclosures).

Consistent with conventional wisdom, a precise accounting report in our model enables an analyst to become more efficient in learning about the quality of voluntarily disclosed information. Indeed, GAAP stipulations have increased over time, and recent regulations call for increased scrutiny by auditors and more oversight by boards. While these regulatory changes likely increase the accuracy of the mandatory financial reports, we show that these changes might actually dampen the frequency of voluntary disclosures. In particular, we use a simple stylized version of the model to show that a more precise accounting report makes voluntary disclosure less likely but any disclosed information more precise. In other words, the (equilibrium) conjecture that analysts can learn the quality of information more precisely makes firms adopt more conservative voluntary disclosure practices.

Next, we model a conservative accounting system that governs mandatory financial reporting as one that underreports favorable news. ${ }^{6}$ That is, when reports are conservative, more favorable news tend to be more representative of firm value, thus, leading analysts to interpret good news more correctly than bad news. This approach is in line with recent analyses that study conservatism (Kwon, 2005; Kwon et al., 2001; Gigler and Hemmer, 2001; Chen et al., 2007). While such conservative accounting systems are arguably desirable in some respects, we show that they could lead to more frequent but less conservative voluntary disclosures in equilibrium. In particular, the threshold quality level, above which hard information is disclosed to the public, is lower for a conservative accounting system. Similarly, under an aggressive accounting system, exactly the opposite happens, i.e. voluntary disclosures are less frequent and more conservative in equilibrium. Thus, our results suggest that efforts to increase precision and encourage desirable biases in mandatory financial reporting can have (unintended) spillover effects on voluntary disclosure.

Our work is related to Verrecchia $(1990,1983)$ which examine issues relating to information quality and discretionary disclosure of information. We build on Verrecchia's work along three dimensions. First, we consider an informational intermediary that strategically learns and gathers information regarding voluntary disclosures. Second, in this paper, the quality (or precision) of voluntary disclosures is endogenously determined in equilibrium and varies across different disclosures. In Verrecchia $(1990,1983)$ the precision of

\footnotetext{
${ }^{4}$ Watts $(2003 \mathrm{a}, \mathrm{b})$ provide a comprehensive discussion of conservatism in accounting.

${ }^{5}$ Our analysis departs from Dye (1985) in that investors are not certain with respect to the quality of information disclosed (quality cannot be credibly disclosed by firms). Other voluntary disclosure models using a structure similar to Dye (1985) include Dye and Sridhar (1995) and Jung and Kwon (1988). Alternatively, other analyses assume that there is (an exogenous) proprietary cost associated with disclosure (see, for example, Dye, 1986). We could have alternatively deployed the disclosure model of Verrecchia (1983) as the basis of our analysis. In particular, extending the work of Verrecchia (1983) to accommodate production of information (e.g. by analysts) in the manner we consider in this paper would have led to qualitatively similar results. In both models introducing an informational intermediary that can follow up after voluntary disclosures will lead to somewhat conservative voluntary disclosure strategies by insiders. For practical reasons, though, we consider a single model of voluntary disclosure.

${ }^{6}$ In the context of mandatory financial reporting, the term "news" refers to what is reported in financial statements, and is not to be confused with the notion of "hard" news disclosed voluntarily.
} 
inside information is exogenously chosen and is the same for all voluntary disclosures. Finally, in our analysis, investors do not know the quality of information received by management, but can only infer its probability distribution in equilibrium. In particular, investors (correctly) infer that the average precision of favorable disclosures is lower relative to that of less favorable disclosures. In contrast, Verrecchia assumes that investors and managers are equally informed regarding the precision of managements' information.

In other related work, Dye and Sridhar (2002) show how capital market reactions to disclosures can guide resource allocation decisions of managers (see also Dye and Sridhar, 1995). While the context is different from ours, a common feature is that both papers take into account the processing of information by capital markets (in our case via the analysts). Dutta and Truman (2002) introduce an element of uncertainty in the information that is voluntarily disclosed, allowing a role for analysts to use their (endowed) private information in interpreting such disclosed information. However, our analysis in this paper is different in that we focus on the costly information production role of the analysts and their ability to strategically scrutinize voluntary disclosures.

The literature linking voluntary and mandatory disclosures is sparse. Fishman and Hagerty (1989) show that more efficient security prices lead to more efficient investment decisions, providing firms with incentives to increase price efficiency through voluntary disclosures. In particular, they show that firms expend more resources to informative disclosures than socially desirable, which is in stark contrast to the notion underlying mandating disclosures that firms tend to underreport. Dye (1990) examines conditions under which voluntary and mandatory disclosures coincide and when mandating disclosures might add social value. However, both these papers do not examine the issue of how certain properties of accounting standards that govern mandatory disclosures (e.g., conservatism) might affect voluntary disclosures.

The paper proceeds as follows. Section 2 describes our model. In Section 3, we derive the basic voluntary disclosure equilibrium. We then introduce the analyst into the model, and derive equilibrium implications for the analyst's role. In Section 4, we present our results on how the nature of accounting standards might influence disclosure precision in equilibrium. Finally, Section 5 concludes.

\section{Model}

Consider a firm with a project that produces a random value of $v$. There are three dates. As in Dye (1985), suppose that in the first period (date 1) management receives a private signal $\langle x, q\rangle$ regarding its value with probability $\lambda \in(0,1)$. The management's signal consists of two components - "hard" information, $x$, and "soft" information, $q$. The manager can credibly disclose "hard" information $x$ (as is typically the case in voluntary disclosure models), but the manager cannot credibly disclose "soft" information $q$. Details about growth in market share relating to the project, about the rollout of products, cash-flow and profitability estimates with respect to the project are all determinants of $x$. Hard information is quantifiable and is something that the management can conveniently communicate. Its disclosure is not mandatory and is subject to the management's discretion.

The implications of the information $x$ for firm value $v$ (e.g., the degree of permanency in growth) are reflected in the manager's "soft" information $q$. This quality dimension is relevant not just to the project under consideration, but affects the returns from existing assets as well. Thus, recent financial reports are a potential source of information for analysts to assess $q$. For our purposes here, we can denote the project (i.e., the firm) by the pair $\langle x, q\rangle$.

The management maximizes the current shareholders' interests in deciding whether to disclose $x$ or not, in date $1 .{ }^{7}$ We consider a simple information structure in which both types of information are equally important, summarized in (1). The random signals $x$ and $q$ are statistically independent and smoothly distributed over their support (with density functions $f$ and $g$, respectively).

$$
v=x q \quad \text { where } x \sim F \in[0, \infty), \quad q \sim G \in[0,1] .
$$

\footnotetext{
${ }^{7}$ As in Dye (1985) we suppose that there is no agency problem between management and current equity holders. Namely, the manager will be paid his marginal product and will adopt any disclosure policy that current shareholders will prefer.
} 
With this structure, it is convenient to think of soft information or $q$ as quality of hard information, or simply, quality of information. In particular, when $q=1, x$ is a precise signal of $v$ and when $q=0$ the disclosure of $x$ does not convey anything about $v$. Outsiders cannot observe whether or not the management has received the signal, neither can the management communicate the receipt of the signal credibly.

Next, we describe the role of analysts as external providers of information. In the second period (date 2), analysts produce a noisy (public) signal regarding firm value, and subsequently the firm's shares trade on the market. Disclosures by firms are news events that trigger information production and analysis by analysts and financial markets (Dye and Sridhar, 2002). Analysts act as "gatekeepers" for the investment community through their information acquisition and processing activities. They evaluate a firm's voluntary disclosure in light of other information available to them from its (mandated) financial statements, and from industry and product market trends. However, information acquisition, processing and analysis is costly. Analysts tend to specialize narrowly in specific industries, and are selective in their choice of which firms to follow and which disclosures to scrutinize - they may not subject good news and bad news disclosures to the same level of scrutiny.

Based on these considerations, we allow the analyst's effort to vary with the level of disclosure, $x$. Specifically, let $\gamma_{x}$ represent the level of the analyst's scrutiny when the firm discloses $x$, where $\gamma_{x} \in[0, \bar{\gamma})$, for some $\bar{\gamma}$ such that $0<\bar{\gamma}<1$. The level of scrutiny $\gamma_{x}$ represents the probability that the analyst will discover $q$ when a firm $\langle x, q\rangle$ discloses $x$, i.e., the analyst discovers the true value $v=x q$. With probability $\left(1-\gamma_{x}\right)$, however, the analyst learns nothing (null).

$$
\text { Analystsignal }= \begin{cases}q, & \gamma_{x}, \\ \text { Null, } & 1-\gamma_{x}\end{cases}
$$

We assume that there is no analyst activity in the absence of disclosure. For example, if a firm withholds information regarding a proposed project it is unlikely that analysts would take the value of this new project into account when analyzing the firm. On the other hand, if there is disclosure about a new project then it is likely that analysts delve into assessing the value of the project. This assumption is in line with the empirical evidence: Bushee and Miller (2006) examine a sample of firms that hire outside investment relations professionals to increase their visibility in the investment community, and provide evidence that analyst following increases following a concurrent increase in disclosure levels for these firms. ${ }^{8}$

The price of the firm at date $2, P_{2}$, equals the (conditional) expectation of firm value $v$, and depends on whether disclosure occurred, and on the informativeness of the analyst's signal. Let $\mathrm{P}(N D)$ be the market price given non-disclosure, $\mathrm{P}(x)$ be the market price following disclosure $x$ when the analyst's signal is not informative, and $v(=x q)$ be the market price following disclosure $x$ (by firm $\langle x, q\rangle$ ) when the analyst's signal is informative. Formally,

$$
\text { Date } 2 \text { price: } \mathrm{P}_{2}= \begin{cases}\mathrm{P}(N D) & \text { if no disclosure, } \\ \mathrm{P}(x) & \text { if disclosure of } x, \text { and analyst's signal not informative, } \\ x q & \text { if disclosure of } x, \text { and analyst's signal is informative. }\end{cases}
$$

Finally, the firm's value $v$ realizes (date 3). The time line is summarized below, and Fig. 1 pictorially depicts the different disclosure/non-disclosure nodes and the corresponding prices.

- Date 1 . The management receives a private signal $\langle x, q\rangle$ with probability $\lambda \in(0,1)$, and decides whether to disclose $x$. Outsiders do not observe whether or not the management has received the signal.

- Date 2. Analysts produce a noisy (public) signal regarding firm value, and the firm's shares trade on the market.

- Date 3. The firm's value $v=x q$ realizes.

\footnotetext{
${ }^{8}$ For our results, however, we need not restrict ourselves to this assumption. In particular, it suffices to assume that voluntary disclosures marginally help analysts assess the value of the firm.
} 


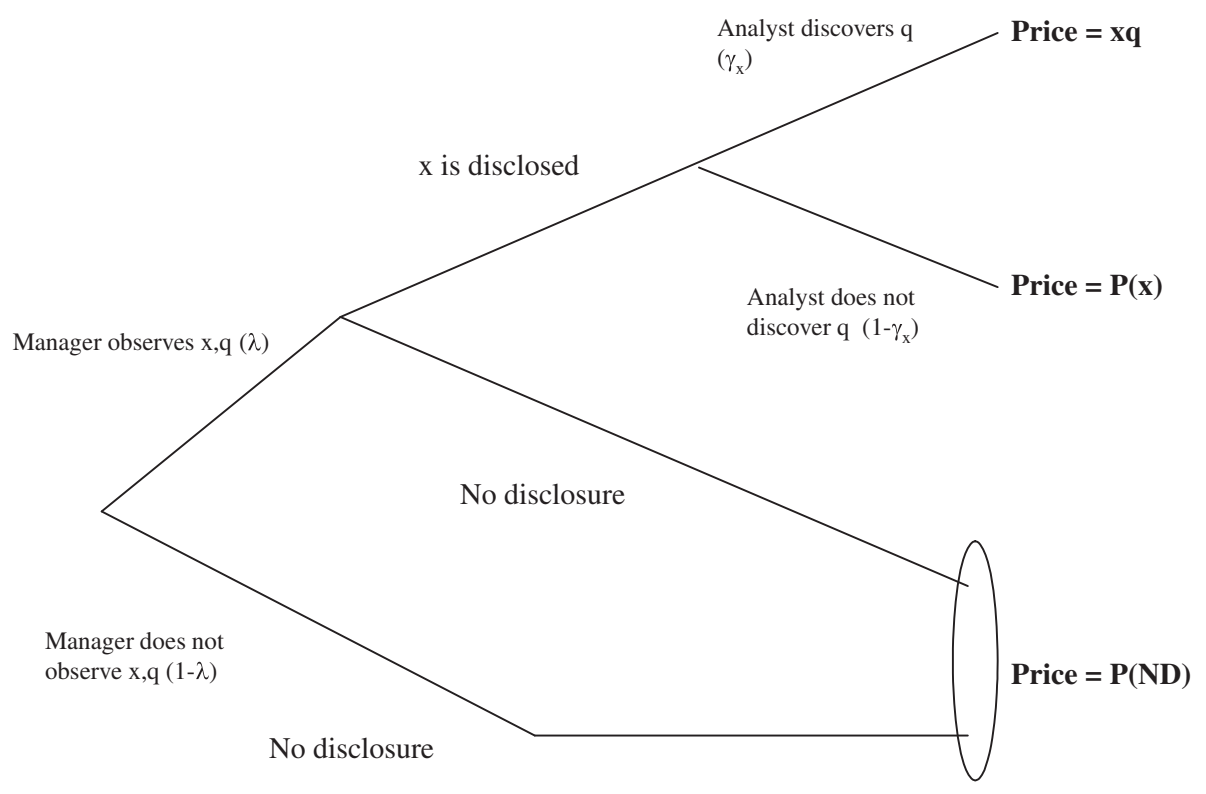

Fig. 1. Disclosure events and prices.

\section{Disclosure equilibrium}

In this section, we derive the basic voluntary disclosure equilibrium for any given level of analyst scrutiny $\left\{\gamma_{x}\right\}_{x \geqslant 0}$. We assume for now that following a disclosure of $x$, outsiders can costlessly determine the nature of soft information $q$ with probability $\gamma_{x}$. This allows us to keep aside information production issues and focus squarely on identifying and establishing a voluntary disclosure equilibrium in terms of quality threshold levels. In the next section, we formally incorporate strategic and costly information production via the analyst's choice of $\gamma_{x}$, and examine the consequent implications for the disclosure equilibrium.

The firm's voluntary disclosure decision is whether to disclose $x$ or not. If it does not disclose, then outsiders cannot tell whether it has knowledge of $\langle x, q\rangle$ but has chosen not to disclose, or whether it does not have knowledge of $\langle x, q\rangle$ (recall that the manager is privately informed with probability $\lambda \in(0,1)$ ). As in voluntary disclosure models, we assume that the firm can disclose $x$ in a credible manner. We assume that the firm's management maximizes the current (i.e., date 2) shareholders' interests in deciding whether to disclose $x$ or not. This is essentially equivalent to maximizing expected price at date $2, P_{2}$. Thus, the manager of firm $\langle x, q\rangle$ will disclose $x$ only if $E\left[P_{2} \mid\right.$ No Disclosure $]$ is smaller than $E\left[P_{2} \mid\right.$ Disclosure of $x$ by firm $\left.\langle x, q\rangle\right]$ where, ${ }^{9}$

$$
\begin{aligned}
& E\left[P_{2} \mid \text { No Disclosure }\right]=\mathrm{P}(N D), \\
& E\left[P_{2} \mid \text { Disclosure of } x \text { by firm }\langle x, q\rangle\right]=\gamma_{x}(x q)+\left(1-\gamma_{x}\right) \mathrm{P}(x) .
\end{aligned}
$$

To define a disclosure equilibrium, it is useful to first characterize the nature of the disclosure regions in any equilibrium. Critical proofs are in Appendix A. ${ }^{10}$

Lemma 1. In any disclosure equilibrium, for any given $\left\{\gamma_{x}\right\}_{x \geqslant 0}$, the disclosure region is "upper-tailed." That is, there exists $\left\{q_{x}\right\}_{x \geqslant 0}$ such that the manager will disclose $x$ only if $q \geqslant q_{x}$, where $q_{x} \in[0,1]$.

This lemma allows us to completely represent a firm's disclosure policy using the disclosure thresholds $\left\{q_{x}\right\}_{x \geqslant 0}$. It is useful to define three possible disclosure regions:

- A non-disclosure region, $N D$, consisting of all $x$ for which the manager never discloses regardless of the value of $q$. That is, $N D=\left\{x \geqslant 0: q_{x}=1\right\}$.

\footnotetext{
${ }^{9}$ Without loss of generality, we assume that when indifferent, the manager will choose disclosure of $x$ over non-disclosure.

${ }^{10}$ Some of the proofs in Appendix A are abbreviated. A detailed version of these proofs are available upon request from the authors.
} 
- A full disclosure region, $D$, consisting of all $x$ for which the manager always discloses regardless of the value of $q$. That is, $D=\left\{x \geqslant 0: q_{x}=0\right\}$.

- A partial disclosure region, $P D$, for which the manager discloses only when $q \geqslant q_{x}$ for some $q_{x} \in(0,1)$. That is, $P D=\left\{x \geqslant 0: q_{x} \in(0,1)\right\}$.

We now define a disclosure equilibrium and show its existence.

Proposition 1 (Disclosure equilibrium). There exists a voluntary disclosure equilibrium consisting of a set of thresholds $\left\{q_{x}\right\}_{x \geqslant 0}$, and a set of prices $\mathrm{P}(N D)$ and $\{\mathrm{P}(x)\}_{x \geqslant 0}$ such that:

I. If the manager learns $\langle x, q\rangle$, then he discloses $x$ only if $q \geqslant q_{x}$, where

$$
q_{x}= \begin{cases}1 & \text { if } \mathrm{P}(N D)-\left(1-\gamma_{x}\right) \mathrm{P}(x) \geqslant \gamma_{x} x, \\ 0 & \text { if } \mathrm{P}(N D)-\left(1-\gamma_{x}\right) \mathrm{P}(x) \leqslant 0, \\ \frac{\mathrm{P}(N D)-\left(1-\gamma_{x}\right) \mathrm{P}(x)}{\gamma_{x} x} & \text { if otherwise. }\end{cases}
$$

II. The market rationally sets prices $\mathrm{P}(N D)$ and $\mathrm{P}(x)$ as ${ }^{11}$

$$
\mathrm{P}(N D)=\psi\left[(1-\lambda) \mathrm{E}(x) \mathrm{E}(q)+\lambda \int_{0}^{\infty} x f(x)\left(\int_{0}^{q_{x}} q g(q) \mathrm{d} q\right) \mathrm{d} x\right],
$$

where

$$
\begin{aligned}
& \psi=\frac{1}{1-\lambda+\lambda \int_{0}^{\infty} G\left(q_{x}\right) f(x) \mathrm{d} x} . \\
& \mathrm{P}(x)= \begin{cases}x \mathrm{E}\left(q \mid q \geqslant q_{x}\right) & \text { for } q_{x} \in[0,1), \\
x & \text { for } q_{x}=1 .\end{cases}
\end{aligned}
$$

Proposition 1 establishes the equilibrium disclosure strategy of the manager, and the interim market prices. The disclosure threshold levels $\left\{q_{x}\right\}_{x \geqslant 0}$ (summarized in (5)) follow from Lemma 1, the expected value of the firm, $v$, conditional on the event of non-disclosure, is given by (6), and the expected value of the firm, conditional on the event of disclosure of $x$ is given by (7). While a detailed proof of Proposition 1 is in Appendix A, we sketch the proof of the existence of an equilibrium here. First, we use (5) and (7) to establish functions $\left\{Q_{x}(\mathrm{P}(N D))\right\}_{x \geqslant 0}$, which yield disclosure thresholds $q_{x}=Q_{x}(\mathrm{P}(N D))$ for any given price associated with non-disclosure $\mathrm{P}(N D)$. Second, we show that the function $Q_{x}(\mathrm{P}(N D))$ is unique, and continuous for any given $x \geqslant 0$. This implies three unique disclosure regions as a function of $\mathrm{P}(N D)$ :

- $N D=\left\{x \geqslant 0: Q_{x}(\mathrm{P}(N D))=1\right\}$.

- $D=\left\{x \geqslant 0: Q_{x}(\mathrm{P}(N D))=0\right\}$.

- $P D=\left\{x \geqslant 0: Q_{x}(\mathrm{P}(N D)) \in(0,1)\right\}$.

Finally, because the price $\mathrm{P}(N D)$ is determined by (6) in equilibrium, we show that there exists a solution $\mathrm{P}(N D)=z^{*}$ satisfying (6) where the threshold $q_{x}$ is given by the function $Q_{x}(\mathrm{P}(N D))$. That is, there exists a solution $z^{*}$ to $\Gamma\left(z^{*}\right)=0$, where

$$
\begin{aligned}
& \Gamma(z) \equiv z-\psi(z)\left[(1-\lambda) \mathrm{E}(x) \mathrm{E}(q)+\lambda \int_{0}^{\infty} x f(x)\left(\int_{0}^{Q_{x}(z)} q g(q) \mathrm{d} q\right) \mathrm{d} x\right], \\
& \text { where } \psi(z)=\frac{1}{1-\lambda+\lambda \int_{0}^{\infty} G\left(Q_{x}(z)\right) f(x) \mathrm{d} x} .
\end{aligned}
$$

\footnotetext{
${ }^{11}$ In the non-disclosure region $\left(q_{x}=1\right)$, the price $\mathrm{P}(x)$ represents an out-of-equilibrium price. The equilibrium is robust to alternative out-of-equilibrium beliefs. See the proof of Proposition 1 for details.
} 
Having established existence of the equilibrium, it is of interest to know whether the non-disclosure, disclosure, or partial disclosure regions are non-empty.

Corollary 1. In equilibrium, the non-disclosure set, $N D$, the full disclosure set, $D$, and the partial disclosure set, $P D$, are non-empty sets in equilibrium, provided that $\gamma_{x} \in[0, \bar{\gamma})$ such that $0<\bar{\gamma}<1$.

Corollary 1 establishes that quality $(q)$ of information $(x)$ determines whether it will be disclosed in equilibrium. This result introduces a natural role for external information production (e.g., by financial analysts). Moreover, the existence of the intermediate region in which the quality threshold levels are strictly interior suggests that analysts might strategically apply different levels of scrutiny to different disclosures. It also suggests that the threshold levels in this partial disclosure region, and the size of this region, depend on the extent to which outsiders can determine the nature of the soft information $q$. Therefore, it is important to examine the disclosure equilibrium in which analysts have a costly information production role. To achieve this end, we incorporate a strategic analyst into our analysis in the next section.

\subsection{The strategic analyst}

In this section, we examine the analyst's decision with respect to the level of scrutiny following a disclosure of $x$, and its implications for the disclosure equilibrium. In modeling the strategic analyst, we assume that analysts care about the accuracy of their forecasts, or, equivalently, about the level of uncertainty about the firm prospects, while taking into account the cost of conducting analysis (see, for example, Mikhail et al., 1999). The more accurate the analyst's signal, the more informative is the share price at date 2 , and the less volatile is the firm's share price between date 2 and date 3 . In particular, let $r_{x}$ be the gross return on the stock of firm $\langle x, q\rangle$ between date 2 and date 3, following disclosure of $x$ ( $r_{x}$ is discussed further below):

$$
r_{x}=\frac{x q}{P_{2}} .
$$

Increasing the accuracy of the analyst's signal, however, requires greater (costly) effort on the part of the analyst. In particular, scrutiny level $\gamma$ imposes a cost on the analyst $c(\gamma)$, with $c(0)=0, c^{\prime}(0)=0, c^{\prime \prime}(\gamma)>0$, and $c^{\prime}(\bar{\gamma})=\infty$ for some $\bar{\gamma} \in(0,1)$. In choosing the level of scrutiny, the analyst will balance the accuracy improvement against the cost of information acquisition and analysis. The analyst's utility, $U_{\mathrm{A}}(x, \gamma)$, following disclosure of $x$ and scrutiny level $\gamma$ is,

$$
U_{\mathrm{A}}(x, \gamma)=-\operatorname{Var}\left(r_{x} \mid \text { Disclosure of } x, \gamma\right)-c(\gamma) .
$$

We now derive the distribution of the return $r_{x}$. Consider firm $\langle x, q\rangle$ that discloses $x$, and consider scrutiny level $\gamma$. Then, with probability $\gamma$ the analyst's signal is accurate, and the date 2 price $P_{2}$ equals the true value of the firm $x q$. In this case, the gross return on the firm's stock is $r_{x}=\frac{x q}{P_{2}}=1$. With probability $1-\gamma$, however, the analyst's signal is not informative, and the date 2 price following disclosure of $x$ is given by $\mathrm{P}(x)$. In this case $r_{x}=\frac{x q}{\mathrm{P}(x)}$. Thus, following disclosure of $x$,

$$
r_{x}= \begin{cases}1 & \text { w.p. } \gamma \\ \frac{x q}{\mathrm{P}(x)} & \text { w.p. } 1-\gamma .\end{cases}
$$

Following disclosure of $x$, the analyst's best response choice of scrutiny, $\gamma_{x}$, to the manager's (cutoff) strategy of disclosing $x$ when $q \geqslant q_{x}$ (for some $q_{x} \in[0,1)$ ) is given by

$$
\gamma_{x} \in \arg \max _{\gamma \leqslant \bar{\gamma}} U_{\mathrm{A}}(x, \gamma)=\arg \min _{\gamma \leqslant \bar{\gamma}} \operatorname{Var}\left(r_{x} \mid x, q \geqslant q_{x}, \gamma\right)+c(\gamma) \text {. }
$$

One can calculate, $\operatorname{Var}\left(r_{x} \mid x, q \geqslant q_{x}, \gamma\right)=(\gamma-1)\left[1-\frac{\mathrm{E}\left(q^{2} \mid q \geqslant q_{x}\right)}{\mathrm{E}^{2}\left(q \mid q \geqslant q_{x}\right.}\right)$, while noting that $\mathrm{P}(x)=x \mathrm{E}\left(q \mid q \geqslant q_{x}\right)$. Thus, the objective function reduces to,

$$
\gamma_{x} \in \arg \min _{\gamma \leqslant \bar{\gamma}}(\gamma-1)\left[1-\frac{\mathrm{E}\left(q^{2} \mid q \geqslant q_{x}\right)}{\mathrm{E}^{2}\left(q \mid q \geqslant q_{x}\right)}\right]+c(\gamma) .
$$


Thus, the analyst's best response level of scrutiny $\gamma_{x}$ given the disclosure of $x$ and threshold level $q_{x}$ is uniquely given by the solution to the following first order condition:

$$
c^{\prime}\left(\gamma_{x}\right)=\frac{\mathrm{E}\left(q^{2} \mid q \geqslant q_{x}\right)}{\mathrm{E}^{2}\left(q \mid q \geqslant q_{x}\right)}-1 .
$$

The second order condition is simply $c^{\prime \prime}\left(\gamma_{x}\right)>0$, which holds given the assumed convexity of $c(\gamma)$. Notice that because $c^{\prime}(\bar{\gamma})=\infty$, the analyst's choice of the level of scrutiny $\gamma_{x}<\bar{\gamma}<1$ for all $x$. To establish the existence of the disclosure equilibrium we assume that the conditional expectation of $q$ exhibits the following property.

Assumption A1. $\frac{\partial \mathrm{E}(q \mid q \geqslant z)}{\partial z}<1$ for $z \in(0,1)$.

This assumption, which has been used in the disclosure literature, ${ }^{12}$ merely requires that the conditional expectation of $q$ varies smoothly and boundedly in the conditioning variable, which commonly used distributions satisfy. ${ }^{13}$ Intuitively, the conditional variance of $q$ decreases in the conditioning variable (i.e., $\operatorname{Var}(q \mid q \geqslant z)$ is decreasing in $z$ for $z \in[0,1))$ for distributions that satisfy Assumption A1.

Lemma 2. Given Assumption $\mathrm{A} 1$, the ratio $\frac{\mathrm{E}\left(q^{2} \mid q \geqslant z\right)}{\mathrm{E}^{2}(q \mid q \geqslant z)}$ is decreasing in $z$, i.e., $\frac{\partial}{\partial z}\left(\frac{\mathrm{E}\left(q^{2} \mid q \geqslant z\right)}{E^{2}(q \mid q \geqslant z)}\right)<0$ for $z \in[0,1) .{ }^{14}$

Proposition 2 formally addresses the existence of a disclosure equilibrium when the analyst strategically chooses the degree of scrutiny $\gamma_{x}$.

Proposition 2 (Disclosure equilibrium with a strategic analyst). Given Assumption A1, there exists a voluntary disclosure equilibrium consisting of a set of thresholds $\left\{q_{x}\right\}_{x \geqslant 0}$, a set of prices $\mathrm{P}(N D)$ and $\{\mathrm{P}(x)\}_{x \geqslant 0}$, and the degree of analyst scrutiny $\left\{\gamma_{x}\right\}_{x \geqslant 0}$ such that:

I. If the manager learns $\langle x, q\rangle$, then he discloses $x$ only if $q \geqslant q_{x}$, where $q_{x}$ is given by (5).

II. The market rationally sets prices $\mathrm{P}(N D)$ as in $(6)$ and $\mathrm{P}(x)$ as in (7).

III. Upon observing $x$ when disclosed, the analyst chooses the degree of scrutiny $\gamma_{x}$ as ${ }^{15}$ :

$$
\gamma_{x}= \begin{cases}c^{\prime-1}\left(\frac{\mathrm{E}\left(q^{2} \mid q \geqslant q_{x}\right)}{\mathrm{E}^{2}\left(q \mid q \geqslant q_{x}\right)}-1\right) & \text { for } q_{x} \in[0,1), \\ 0 & \text { for } q_{x}=1 .\end{cases}
$$

Having shown the existence, we examine certain properties of the disclosure equilibrium. For the rest of the paper, we analyze and extend the equilibrium identified in Proposition 2, and thus implicitly invoke Assumption A1 throughout. To begin with, it is interesting to investigate how the analyst responds to the equilibrium disclosures, i.e., when the analyst would choose to engage in closer scrutiny and when not. The following corollary provides an important insight in this respect.

Corollary 2. Consider any two values of disclosed information $x_{1}, x_{2}$ such that $x_{1} \neq x_{2}$. If, in equilibrium, $q_{x_{1}}<(=) q_{x_{2}}$, then it must be that $\gamma_{x_{1}}>(=) \gamma_{x_{2}}$.

A lower threshold implies that the disclosure is more imprecise because the range of possible $q$ values expands. The above result therefore implies that analysts find it more rewarding to investigate more imprecise disclosures. This is intuitively appealing because one would expect the analyst's information gathering role to be of greater value when disclosures are less precise.

We next characterize the nature of the various disclosure regions in equilibrium.

\footnotetext{
${ }^{12}$ See, for example, footnotes 21 and 22 in Dye (1986).

${ }^{13}$ Distributions satisfying this property include those that exhibit increasing failure rates (IFR), i.e., $\frac{\partial}{\partial t} \frac{g(t)}{1-G(t)} \geqslant 0$. This is a large family of distributions that includes for example the Uniform, Exponential, and special cases of Gamma and Weibull. Moreover, one can show that for any positive (and continuous) IFR random variable $Y$, the conditional random variable $Y \mid Y \leqslant 1$ is also IFR.

${ }^{14}$ For the sake of brevity, we do not offer a proof of Lemma 2, but will be glad to provide it upon request.

${ }^{15}$ In the non-disclosure region (i.e., $x$ for which $q_{x}=1$ ), $\gamma_{x}$ represents an out-of-equilibrium level of scrutiny. This out-of-equilibrium specification is in keeping with the specification for $\mathrm{P}(x)$ for $x \in N D$. See the proof of Proposition 2 for details.
} 
Corollary 3. In the disclosure equilibrium with a strategic analyst, the non-disclosure set ND is not empty. In particular, disclosure will not take place (i.e., $q_{x}=1$ ) if and only if $x \leqslant \mathrm{P}(N D)$.

Intuitively, for the manager of a firm $\langle x, q\rangle$ to disclose information $x$, it must be that either the true value of the firm is larger than the value of a non-disclosing firm (i.e., $x q \geqslant \mathrm{P}(N D)$ ), or that the markets' valuation following disclosure (which might not represent the true value of the firm) is larger than the value of a nondisclosing firm $\mathrm{P}(x) \geqslant \mathrm{P}(N D)$. In either case $x>\mathrm{P}(N D)$, as an upper bound for $\mathrm{P}(x)$ is $x$. Thus, the manager of the firm $\langle x, q\rangle$, where $x \leqslant \mathrm{P}(N D)$, will not benefit from disclosure. If $x>\mathrm{P}(N D)$, however, then we must expect managers to disclose even though there information is not precise (i.e., $q_{x}<1$ ). Clearly, if market participants believe that managers only disclose information when information is precise, then (in equilibrium) the market should price securities appropriately, i.e., $\mathrm{P}(x)=x>\mathrm{P}(N D)$. However, given these beliefs, managers would benefit (in some cases) by disclosing even when their information is not precise, and this would not be an equilibrium. Thus, disclosure of imprecise information will take place in equilibrium whenever $x>\mathrm{P}(N D)$, and the non-disclosure region is not degenerate (recall that $\mathrm{P}(N D)>0$ from (6)).

Next, we examine whether there would exist a region of $x$ in which the firm always discloses (i.e., $q_{x}=0$ ).

Corollary 4. In the disclosure equilibrium with a strategic analyst, the disclosure set $D$ is not empty. In particular, there exists a finite $\bar{x}$ such that disclosure occurs regardless of the value of $q$ if and only if $x \geqslant \bar{x}\left(i . e ., q_{x}=0\right)$.

Because managers desire to maximize expected stock price $P_{2}$ (at date 2), they have a natural incentive to disclose favorable hard information, all else equal. This incentive is somewhat muted by the level of scrutiny adopted by rational analysts anticipating such behavior by managers. Nevertheless, Corollary 4 establishes that in equilibrium, there would exist a point beyond which the hard information $x$ is sufficiently "good" that managers will benefit from its disclosure regardless of its quality and despite analyst scrutiny.

Given Corollaries 3 and 4, we can now identify an intermediate region in which managers conservatively disclose information. In this partial disclosure region, disclosure occurs only if $q \in\left(q_{x}, 1\right]$ for $q_{x} \in(0,1)$.

Corollary 5. In the disclosure equilibrium with a strategic analyst, the partial disclosure set $P D=(\mathrm{P}(N D), \bar{x})$ is not empty.

Since manager's disclosure decision depends on $x$, a question that naturally arises is whether, in equilibrium, there exists a (monotonic) relation between the threshold quality level $q_{x}$ and the information $x$.

Proposition 3 (Disclosure informativeness with a strategic analyst). In equilibrium, disclosure of bad news is more informative than disclosure of good news. Formally, if $x_{1}>x_{2}$, then $q_{x_{1}} \leqslant q_{x_{2}}$. Moreover, if $x_{1} \in P D$ or $x_{2} \in P D$, and $x_{1}>x_{2}$, then $q_{x_{1}}<q_{x_{2}}$.

Proposition 3 implies that disclosure of good information (high $x$ 's) occurs over a larger quality range notwithstanding the strategic choice of the scrutiny level by the analyst, making the disclosure of favorable hard information less informative relative to unfavorable hard information.

Proposition 3 receives direct empirical support from Kothari et al. (2005). They examine a sample of (voluntary) management forecasts, and conclude that firms tend to withhold bad news forecasts relative to good news forecasts. Moreover, our model predicts that managers tend to disclose bad news, only when it is relatively accurate (i.e., the threshold $q_{x}$ is relatively high for low realizations of $x$ ). Indeed, Kothari et al. (2005) find that the market reaction to the release of bad news (management) forecasts is more pronounced than the market reaction to good news forecasts, evidence that is consistent with this result.

Corollaries 3-5 and Proposition 3 yield a complete characterization of how the equilibrium threshold quality level varies with information that is the subject of disclosure. Fig. 2 presents this characterization pictorially.

Thus, while managers are inherently conservative in disclosing information, Proposition 3 essentially implies that managers are less conservative in disclosing favorable information relative to less favorable information. That is, they are more likely to disclose good information (higher values of $x$ ) and less likely to disclose bad information, as the threshold quality level is lower (higher) for higher (lower) values of $x$.

Since, in equilibrium, good news disclosures are on average more imprecise from the market's standpoint (Proposition 3), it appears reasonable to expect that the analyst can contribute a lot more to minimizing return 


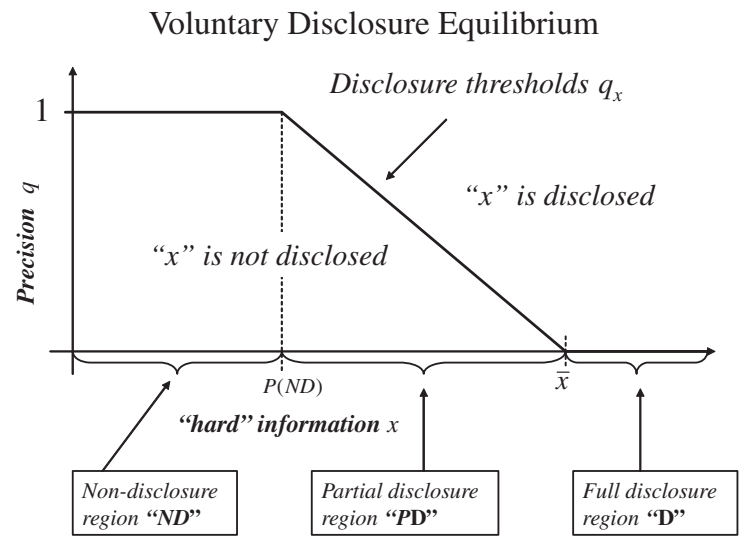

Fig. 2. Voluntary disclosure equilibrium.

variance by choosing higher levels of scrutiny when the firm discloses good information (i.e., for higher values of $x$ ). This intuition underlies the following proposition.

Proposition 4 (Analyst scrutiny). In equilibrium, disclosures of bad news are followed with less scrutiny by analysts relative to disclosures of good news. Formally, if $x_{1}>x_{2}$, then $\gamma_{x_{1}} \geqslant \gamma_{x_{2}}$. Moreover, if $x_{1} \in P D$ or $x_{2} \in P D$, and $x_{1}>x_{2}$, then $\gamma_{x_{1}}>\gamma_{x_{2}}$.

This result follows from Corollary 2 and Proposition 3. The notion that good news disclosures attract greater following among analysts also has empirical support. For example, McNichols and O'Brien (1997) provide evidence that analysts tend to follow stocks about which they have favorable news, and drop coverage of stocks about which they have unfavorable news.

We complete this section with an examination of the relation between disclosure and stock prices. The following proposition characterizes the stock price effect in equilibrium.

Proposition 5 (Stock price response to disclosure). Disclosures of good news result in higher stock prices relative to disclosures of bad news. Formally, $\mathrm{P}(x)$ is increasing in $x$, for $x \in P D \cup D$.

Intuitively, we would expect that the stock price would be higher for good news disclosures. Given our analysis above, however, it is not immediately clear that this would be the case because it does not depend only on $x$, the information disclosed, but also on the equilibrium precision of the disclosure. A higher value of $x$ represents good news for any given level of soft information $q$. But, as we know from Proposition 3 , the manager is willing to lower the threshold level of disclosure for higher values of $x$, making good news disclosures more imprecise. This, in turn, reduces the value of good news disclosures. In equilibrium, however, it does not make sense for an expected price maximizing manager to lower disclosure thresholds for good news disclosures unless the net effect on stock price is positive. This intuition is borne out by Proposition 5 .

\section{Extension: reporting systems and voluntary disclosure}

In the previous section, we demonstrated that the equilibrium content of voluntary disclosures (e.g., disclosures in the MD\&A section) depends on how financial analysts respond to them. In particular, their discovery of $q$ plays a crucial role in equilibrium. In the process of gathering and interpreting information about $q$, analysts presumably rely on the firm's financial statements, those of other firms in the same industry, and news regarding the economy as a whole. This points to a crucial link between the quality of mandated financial reports and firms' voluntary disclosure practices.

Several additions to GAAP have been made since the Securities and Exchange Act of 1934 in response to investors' (and issuers') demand for transparent financial markets, e.g. the recent Sarbanes Oxley Act of 2002. Reforms typically introduce new rules to modify the content of, and the practices that bring about, firms' mandated financial reports. While these reforms have improved the transparency of mandatory financial reports, they apply less to voluntary disclosures. Arguably, both channels of information are of great 
importance to financial markets, as evident from well documented stock price reactions to both types of disclosures. As analysts refer to firms' mandated financial reports in interpreting the quality of voluntary disclosures, we can expect the precision of these financial reports and any biases therein to influence analysts' assessments. This, in turn, would have implications for firms' voluntary disclosure policies. For instance, do more precise mandated financial statements necessarily imply more precise voluntary disclosures? And, does a bias, toward conservatism (aggressiveness), in mandated financial reports imply more conservative (aggressive) voluntary disclosures? We address these questions in the analysis below by incorporating an accounting system and its features into our model in a simple manner.

\subsection{Precision of reporting systems and voluntary disclosure}

Financial reports presumably become more accurate as a result of better external auditing practices, CEO certification, and better board composition, as intended by the Sarbanes Oxley Act. This, in turn, leads to an improvement in analysts' ability to accurately interpret voluntary disclosures. Consider, for example, a disclosure regarding an increase in market share, the quality of which cannot be credibly disclosed and is captured in our model by $q$. The value of $q$ may depend on several things; in particular, it may depend on the firm's ability to sustain profit margins, which an analysis of mandated financial reports might reveal. A precise accounting system will report the firms' earnings in an accurate manner leading to better estimates of profit margins and their persistence, i.e. leading to a more accurate assessment of $q$. Thus, we assume that analysts unearth the true value of $q$, with a higher probability under a more precise accounting system:

$$
\text { Analyst signal }=\left\{\begin{array}{ll}
v, & \gamma+\theta \\
N A, & 1-\gamma-\theta
\end{array} \quad \text { where } \theta \in[0, \bar{\theta}) \text { for some } \bar{\theta} \in(0,1-\bar{\gamma}) .\right.
$$

With this structure we can classify accounting systems in terms of their impact on the analyst's signal as follows:

Classification of accounting systems: Consider accounting systems $A$ and $B$ characterized by $\theta_{A}$ and $\theta_{B}$. The system $A$ is said to be more precise than the system $B$ if $\theta_{A}>\theta_{B}$.

In other words, as the precision of financial reports improves (i.e., $\theta_{A}>\theta_{B}$ ), the greater will be its usefulness to the analyst in discovering $q$.

We next analyze the equilibrium under an accounting system $\theta$. Due to the additive nature of the probability of discovery by the analyst, the (best response) level of scrutiny applied to a disclosure of $x$ (under beliefs that disclosure of $x$ takes place only for $q \geqslant q_{x}$ ) is not affected by the consideration of this more general framework. To examine the analyst's choice of the level of scrutiny in equilibrium, we can calculate the return $r_{x}$ as (recall that $\left.\gamma_{x}+\theta<\bar{\gamma}+\bar{\theta}<1\right)$,

$$
r_{x}= \begin{cases}1 & \text { w.p. } \gamma_{x}+\theta \\ \frac{x q}{\mathrm{P}(x)} & \text { w.p. } 1-\gamma_{x}-\theta\end{cases}
$$

The analyst's problem can be rewritten as $\left(q_{x} \in[0,1)\right)$,

$$
\min _{\gamma \leqslant \bar{\gamma}}(\gamma+\theta-1)\left[1-\frac{\mathrm{E}\left(q^{2} \mid q \geqslant q_{x}\right)}{\mathrm{E}^{2}\left(q \mid q \geqslant q_{x}\right)}\right]+c(\gamma) .
$$

The (best response) level of scrutiny is given by (11), as before. In Proposition 7 (in Appendix A) we recast the disclosure equilibrium of Proposition 2 in the context of an accounting system $\theta$. The difference lies in the manager's best response disclosure strategy. In particular, if the manager learns $\langle x, q\rangle$, then he discloses $x$ only if $q \geqslant q_{x}$, where

$$
q_{x}= \begin{cases}1 & \text { if } \mathrm{P}(N D)-\left(1-\gamma_{x}-\theta\right) \mathrm{P}(x) \geqslant\left(\gamma_{x}+\theta\right) x, \\ 0 & \text { if } \mathrm{P}(N D)-\left(1-\gamma_{x}-\theta\right) \mathrm{P}(x) \leqslant 0, \\ \frac{\mathrm{P}(N D)-\left(1-\gamma_{x}-\theta\right) \mathrm{P}(x)}{\left(\gamma_{x}+\theta\right) x} & \text { if otherwise. }\end{cases}
$$


The equilibrium with an accounting system characterized by $\theta$ exhibits (qualitatively) the same properties as the equilibrium in Proposition 2.

Corollary 6. In any disclosure equilibrium with a strategic analyst under an accounting system $\theta$, Corollaries 2-5 and Propositions 3-5 hold.

We now turn to explore the implications of specific characteristics of accounting systems on the voluntary disclosure equilibrium. For analytical tractability, we examine a setting in which the space of possible outcomes (i.e., "hard" information, $x$ ) is binary.

\subsubsection{An example}

We consider an example in which there are only two levels of hard information. In particular, $x$ is either 1 with probability $p$ or 0 with probability $1-p$, for some $p \in(0,1)$. The value of soft information $q$ is Uniformly distributed over the unit interval, $q \sim \mathrm{U}(0,1)$. We assume that the analyst's cost function is given by $c(\gamma)=\frac{c}{2} \gamma^{2}$, for some $c>0$, and $\gamma \geqslant 0$. In order to simplify the analysis, insure that the equilibrium is unique, and insure that $\gamma_{x}+\theta \in[0,1]$ in equilibrium, we will hereafter consider only $c>\underline{c}=\frac{1}{3(1-\theta)}$ (for some $\theta \in(0,1)$ ). Finally, we restrict attention to the case $\lambda \leqslant \frac{1}{2}$ (which is a sufficient condition for the equilibrium to be unique). The following proposition describes the disclosure equilibrium in this example. ${ }^{16}$

Proposition 7. For $c>\underline{c}=\frac{1}{3(1-\theta)}$, and $\lambda \leqslant \frac{1}{2}$, there exists a unique equilibrium with the following disclosure thresholds:

1. The manager never discloses when $x=0\left(q_{0}=1\right)$.

2. The manager will always disclose when $x=1$ (i.e., $\left.q_{1}=0\right)$ if $c \geqslant \bar{c}$, where $\bar{c}=\frac{1}{3}\left(1-\theta-p \frac{1-\lambda}{1-\lambda+\lambda(1-p)}\right)^{-1}$.

3. The manager will disclose whenever $q \geqslant q_{1}$ with $q_{1} \in\left(0, \frac{1}{2}\right)$ if $c \in(\underline{c}, \bar{c})$.

That is, unfavorable hard news (i.e., $x=0$ ) will never be disclosed regardless of $q$ (i.e., $q_{0}=1$ ). On the other hand, the precision and the frequency of favorable hard news disclosures (i.e., $x=1$ ) depend on analyst scrutiny. When information production is costly to the analyst $(c \geqslant \bar{c})$, then favorable hard news (i.e., $x=1)$ is disclosed regardless of the level of $q$, i.e., threshold $q_{1}$ equals zero, and therefore its precision is the lowest. In contrast, when information production is less costly to the analyst $(c<\bar{c})$, the level of scrutiny deployed by the analyst deters imprecise disclosures. Managers become more conservative by disclosing favorable hard news only when sufficiently accurate, i.e., beyond a strictly positive threshold $q_{1}$. Thus, disclosures are more precise but less frequent.

Having established a unique equilibrium, we analyze the implications of the precision of an accounting system for the voluntary disclosure equilibrium. The following proposition addresses the impact of a marginal increase in the level of precision $\theta$ on the equilibrium threshold level for good news disclosure.

Proposition 8. An increase in the precision of the accounting system leads to more precise (and less frequent) voluntary disclosures of the high outcome $x=1$. Formally, the threshold $q_{1}$ is increasing in $\theta$.

Intuitively, as the precision of the accounting system increases, the probability of discovery of $q$ goes up for any given level of analyst scrutiny. Consequently, the marginal disclosing firm benefits less from disclosure, driving the quality threshold up. And, as the quality threshold increases, there is less demand for analyst scrutiny, all else equal. The following corollary confirms this intuition.

Corollary 7. An increase in the precision of the accounting system leads the analyst to apply less scrutiny following disclosures of the high outcome $x=1$. Formally, the level of analyst scrutiny $\gamma_{1}$ is decreasing in $\theta$.

Proposition 8 and Corollary 7 imply that an increase in the precision of the accounting system affects voluntary disclosure and the role of external information production by the analyst in two important ways. One, voluntary disclosures become more accurate, on average, and are followed with less scrutiny by analysts.

\footnotetext{
${ }^{16}$ The detailed proofs of results relating to the example are omitted from Appendix A for space considerations, but are available upon request from the authors.
} 
Two, voluntary disclosures become less frequent (because of the higher disclosure threshold). Thus, the equilibrium reflects a trade-off between achieving transparency via more frequent (or possibly more timely) voluntary disclosures versus achieving transparency via more precise voluntary disclosures.

An increase in the precision of the accounting system also has an interesting implication for the market response to disclosure. In the example, the ex ante value of the firm, $\mathrm{E}(v)=\frac{p}{2}$, prior to the information event, is independent of the level of precision of the accounting system. The market reactions, however, to disclosures (or silence) are affected by the precision of the accounting system. According to Proposition 8, when the accounting system is more precise voluntary disclosures are also more precise. As a result, the market reaction to a good news disclosure is stronger, i.e., $\mathrm{P}(1)-\mathrm{E}(v)$ is increasing in $\theta$. This is formally stated in Corollary 8 .

Corollary 8. The market reaction to disclosure, $\mathrm{P}(1)-\mathrm{E}(v)$, is increasing in $\theta$.

\subsection{Bias in reporting systems and voluntary disclosure}

The notion of conservatism has received considerable attention in the accounting literature (Watts, 2003a, b). It is often argued that managers, when evaluated on earnings performance, have a tendency to over-report earnings, or more generally, to inflate good news and withhold bad news (e.g., Narayanan, 1985; Dye, 1988; Stein, 1989). It is also argued that a bias toward conservatism in accounting standards has emerged as a natural regulatory response (Watts, 2003a). Instances of conservatism in accounting standards include emphasis on more immediate recognition of expenses when associated revenues are uncertain, on more immediate recognition of losses that are not as yet realized, and delaying recognition of gains till they are realized.

In essence, conservatism systems are more likely to be correct when reporting bad news than when reporting good news (Kwon, 2005; Kwon et al., 2001; Raghu, 2004). Stated differently, conservative systems are less likely to misreport bad news as good news. Consequently, when a sufficiently conservative system does report good news, then the posterior probability that the true state is good is also higher (i.e., the posterior probability that the true state is good given good reported news is higher, the greater the degree of conservatism). ${ }^{17}$

For our purpose here, we take this characteristic of a conservative bias in reporting systems as a given and examine its impact on the voluntary disclosure equilibrium via the analyst's discovery of $q$. In particular, to the extent that analysts depend on financial reports to guide their analysis of firm performance, analysts are less likely to be misled in analyzing them when conservative reporting systems provide good news disclosures, and, therefore, more likely to discover $q$. We use the following structure to capture this intuition:

$$
\begin{aligned}
& \text { Analyst signal }=\left\{\begin{array}{ll}
v, & \gamma+\theta(q) \\
N A, & 1-\gamma-\theta(q)
\end{array} \text { where } \theta:[0,1] \rightarrow[0, \bar{\theta})\right. \\
& \text { for some } \bar{\theta} \in(0,1-\bar{\gamma}) .
\end{aligned}
$$

Recall that higher values of the "soft" information $q$ casts the accompanying "hard" information in favorable light $-q$ represents notions such as sustainability of growth in earnings, market share, etc. To the extent that some of these considerations influence what is reported in financial reports as well, higher values of $q$ will also cast financial reports in favorable light. And, under a conservative accounting system, since financial reports convey "good" news more accurately than "bad" news, analysts are more likely to accurately assess the quality dimension $q$ given "good" news, and are more likely to accurately assess the new project. That is, the probability of the discovery of $q$ increases in $q$ (i.e., $\theta(q)$ is increasing in $q$ ) under a conservative accounting system. ${ }^{18}$ With this structure we can classify biased accounting systems based on their impact on the analyst's signal as follows:

\footnotetext{
${ }^{17}$ Consider, for example, a setting in which the outcome $y \in\{L, H\}, H>L$, and a reporting system that produces a report $r$ based on this outcome, with $r \in\{L, H\}$ such that $\operatorname{Pr}(r=L \mid y=L)=p, \operatorname{Pr}(r=H \mid y=H)=q$. Then a conservative reporting system is one for which $p>q$ (Kwon et al., 2001). It can be shown that as the degree of conservative bias increases in a system ( $p$ in their paper), the posterior probability $\operatorname{Pr}(y=H \mid r=H)$ increases. That is, upon observing $r=H$, inference that the true state is $H$ improves. Similarly, Raghu (2004) intuitively explains that a high grade that is granted by a university with a conservative grading policy is more informative to recruiters, than a high grade otherwise.

${ }^{18}$ The function $\theta(q)$ is continuous.
} 
Bias in accounting systems: An accounting system $A$, characterized by the function $\theta_{A}(q)$, is unbiased if there exists $\theta \geqslant 0$ such that $\theta_{A}(q)=\theta$ (for all $q$ ), and is conservative (aggressive) if $\theta_{A}(q)$ is strictly increasing (decreasing) in $q$.

Lemma 3 in Appendix A (offered without proof) establishes that the equilibrium under a biased accounting system is similar to that stated in Proposition 7 provided Assumption A2 (below) that the function $\theta(q)$ has a bounded derivative - or loosely speaking, as long as any local bias in the accounting system is not "too" extreme.

Assumption A2. The function $\theta:[0,1] \rightarrow[0, \bar{\theta})$ satisfies $\theta(z)>\left|\theta^{\prime}(z)\right|$ for all $z \in[0,1]$.

For the remaining analysis involving bias in accounting systems, we invoke this assumption. We return now to the simple binary example introduced in the previous section in order to analyze the implications of a biased accounting system for the voluntary disclosure equilibrium. It is convenient to consider the following accounting system $\theta(q)$ that allows a clear distinction between "accuracy" and "bias,"

$$
\theta(q)=\theta^{0}+b\left(q-\frac{1}{2}\right)
$$

The parameter $\theta^{0}$ reflects the average level of precision, $E[\theta(q)]=\theta^{0}$. The sign of $b$ reflects the nature of bias: if $b=0$ then the system is unbiased, if $b>0$ then the system is conservative $(\theta(q)$ is increasing in $q)$, and if $b<0$ then the system is aggressive $(\theta(q)$ is decreasing in $q)$. Moreover, an increase in $|b|$ exacerbates the impact of the bias.

Lemma 4 in Appendix A (offered without proof) establishes conditions under which there exists an equilibrium under accounting system $\theta(q)$ similar to that derived in Proposition 6 . The following proposition (offered without proof) describes the equilibrium implications of a marginal increase in the bias parameter $b .{ }^{19}$

Proposition 9. A marginal increase in the level of conservatism, $b$, will lead to more aggressive voluntary disclosures, i.e., lower $q_{1}$. Moreover, the level of analyst scrutiny following good news disclosure $\gamma_{1}$ is increasing in $b$. That is, the analyst will apply less scrutiny when the accounting system is more conservative.

The intuition is as follows. A biased accounting system that communicates favorable soft information more accurately than unfavorable soft information (an asymmetry believed to be a fundamental property of conservatism) has a direct effect on voluntary disclosures. Recall from Proposition 7 that the threshold $q_{1}$ corresponding to $x=1$ is such that $q_{1} \in\left(0, \frac{1}{2}\right)$. Now, consider the marginal firm that is indifferent between disclosure and non-disclosure (i.e., $x=1$ and $q=q_{1}$ ). Since a marginal increase in $b$ decreases the probability of discovery for that firm, it strictly prefers disclosure under a higher $b$-driving down the equilibrium disclosure threshold (i.e., $q_{1}$ is decreasing in $b$ ).

\section{Discussion and conclusion}

In this paper, we introduce an accuracy-enhancing analyst as an information intermediary between the firm and the market and examine the manner in which the analyst scrutinizes and processes disclosures, and the impact on the voluntary disclosure equilibrium. Traditional voluntary disclosure models assume that whenever firms disclose they disclose truthfully, leaving no room for any additional interpretation and analysis. To create a role for the analyst, we build on Dye (1985) and analyze a model in which the information asymmetry between the firm and outsiders consists of two components - one that is quantifiable and disclosable (hard information), and the other that is hard to quantify (soft information or the quality dimension).

Analysis of our disclosure equilibrium yields three main results. First, bad news disclosures are more informative (or precise) than good news disclosures, a result that is consistent with the empirical evidence in Kothari et al. (2005). Second, analysts are sceptical regarding good news disclosures and respond by increasing the level of scrutiny. This result is in keeping with the empirical evidence in McNichols and O'Brien (1997) suggesting that analysts tend to follow stocks about which they have favorable news, and drop coverage on

\footnotetext{
${ }^{19}$ The proofs for Lemmas 3 and 4 and Proposition 9 are available from the authors upon request.
} 
stocks about which they have unfavorable news. Third, despite being relatively uninformative, good news disclosures are associated with higher stock prices than bad news disclosures.

By admitting a role of the analyst, our model also provides us with an opportunity to forge a link, albeit an indirect one, between mandatory and voluntary disclosures. In particular, mandatory financial reports likely provide analysts with an important source of information in scrutinizing the quality of accompanying voluntary disclosures (such as disclosures in MD\&A sections of financial reports) or voluntary disclosures that follow the publication of financial reports. Consequently, the precision of financial reports and any biases in these reports are likely to influence their judgments as well, which, in turn, are likely to affect the efficacy of voluntary disclosures. Indeed, the slant toward conservatism in accounting is well known and widely debated.

Using a stylized binary setting, we show that an increase in the precision of financial reports results in less frequent yet more precise voluntary disclosures, and lower levels of scrutiny by analysts following voluntary disclosures. We also show that a conservative bias in accounting reports could lower equilibrium quality thresholds with respect to voluntary disclosures, resulting in more frequent yet less informative (or less precise) disclosures. These results suggest a trade-off in achieving transparency between the frequency and precision of voluntary disclosures.

To the best of our knowledge, this paper is among the first to explicitly examine the impact of the information intermediary role of an analyst on voluntary disclosures. We also take a first step in examining how known biases in financial reporting can have spillover effects on informativeness of voluntary disclosures. A limitation of our approach in this respect is the exogenous manner in which we have modeled conservatism - clearly an avenue for future research.

\section{Appendix A}

Proof of Lemma 1. The manager of firm $\langle x, q\rangle$ will act to maximize the expected (interim) market price, $P_{2}$, and will disclose information $x$ if and only if $E\left[P_{2} \mid\right.$ Disclosure of $x$ by firm $\left.\langle x, q\rangle\right] \geqslant \mathrm{P}(N D)$ where $E\left[P_{2} \mid\right.$ Disclosure of $x$ by firm $\left.\langle x, q\rangle\right]=\gamma_{x}(x q)+\left(1-\gamma_{x}\right) \mathrm{P}(x)$ (see (4)). Let $\Theta_{x} \subseteq[0,1]$ denote the disclosure set, in terms of $q$, for a given $x$. Thus, $\Theta_{x}=\left\{q \in[0,1] \mid \gamma_{x} x q+\left(1-\gamma_{x}\right) \mathrm{P}(x) \geqslant \mathrm{P}(N D)\right\}$. Clearly, if $q^{0} \in \Theta_{x}$ for some $q^{0} \in[0,1)$, then for any $q^{\prime} \in\left(q^{0}, 1\right], q^{\prime} \in \Theta_{x}$. This establishes that if $\Theta_{x}$ is not an empty set, then it must be of the form $\Theta_{x}=\left[q_{x}, 1\right]$ for some $q_{x} \in[0,1)$. On the other hand, if $\Theta_{x}$ is an empty set then without loss of generality we can write $\Theta_{x}=\left[q_{x}, 1\right]$ for $q_{x}=1$ (recall that the cumulative distribution function $G(\cdot$ ) is continuous, and $G(1)=1$ ). Thus, if an equilibrium exists, the equilibrium disclosure region will be "upper-tailed".

The next two lemmas are not referred to in the body of the text but facilitate the proofs of Propositions 1 and 2.

Lemma A1. Given the disclosure threshold levels $\left\{q_{x}\right\}_{x \geqslant 0}$, the market price following a disclosure of $x$ is $\mathrm{P}(x)=$ $x \mathrm{E}\left(q \mid q \geqslant q_{x}\right)$ for $q_{x} \in[0,1)$, and the market price following non-disclosure, $\mathrm{P}(N D)$, is given by,

$$
\begin{aligned}
& \mathrm{P}(N D)=\psi\left[(1-\lambda) \mathrm{E}(x) \mathrm{E}(q)+\lambda \int_{0}^{\infty} x f(x)\left(\int_{0}^{q_{x}} q g(q) \mathrm{d} q\right) \mathrm{d} x\right], \\
& \text { where } \psi=\frac{1}{1-\lambda+\lambda \int_{0}^{\infty} G\left(q_{x}\right) f(x) \mathrm{d} x} .
\end{aligned}
$$

Proof of Lemma A1. Consider the market price following a disclosure of $x$ (for which $q_{x} \in[0,1)$ ). The expected value of the firm conditional on disclosure of $x$ is $\mathrm{E}(\tilde{x} \tilde{q} \mid D)=\mathrm{E}\left(\tilde{x} \tilde{q} \mid \tilde{x}=x, \tilde{q} \geqslant q_{x}\right)=x \mathrm{E}\left(\tilde{q} \mid \tilde{q} \geqslant q_{x}\right)$, where $x$ denotes a specific realization of this random variable $\tilde{x}$.

Consider next the market price following non-disclosure, $\mathrm{P}(N D)=\mathrm{E}(\tilde{x} \tilde{q} \mid N D)$. For convenience let $Y \in\{0,1\}$, with $Y=0$ (1) represent the state in which the manager is uninformed (informed), respectively. Thus, $\mathrm{P}(Y=0)=1-\lambda$. We can calculate the expectation $\mathrm{E}(\tilde{x} \tilde{q} \mid N D)$ as

$$
\mathrm{P}(N D)=\mathrm{E}(\tilde{x} \tilde{q} \mid N D)=\mathrm{E}_{y}(\mathrm{E}(\tilde{x} \tilde{q} \mid N D, Y) \mid N D)=\sum_{y \in\{0,1\}} \mathrm{E}(\tilde{x} \tilde{q} \mid N D, Y=y) P(Y=y \mid N D) .
$$


The joint probabilities of non-disclosure and $Y$ are $\mathrm{P}(Y=0, N D)=1-\lambda, \mathrm{P}(Y=1, N D)=\lambda \int_{0}^{\infty} G\left(q_{z}\right) f(z) \mathrm{d} z$, and the probability of non-disclosure is $\mathrm{P}(N D)=1-\lambda+\lambda \int_{0}^{\infty} G\left(q_{z}\right) f(z) \mathrm{d} z$. We can therefore calculate the posterior probability of the manager being informed, $Y=1$ conditional on non-disclosure as

$$
\mathrm{P}(Y=1 \mid N D) \equiv \hat{\lambda}=\frac{\mathrm{P}(Y=1, N D)}{\mathrm{P}(Y=0, N D)+\mathrm{P}(Y=1, N D)}=\frac{\lambda \int_{0}^{\infty} G\left(q_{z}\right) f(z) \mathrm{d} z}{1-\lambda+\lambda \int_{0}^{\infty} G\left(q_{z}\right) f(z) \mathrm{d} z} .
$$

The expected value of the firm conditional on non-disclosure by an uninformed manager is $\mathrm{E}(\tilde{x} \tilde{q} \mid$ $N D, Y=0)=\mathrm{E}(x) \mathrm{E}(q)$. To calculate the expectation $\mathrm{E}(\tilde{x} \tilde{q} \mid N D, Y=1)$, first note that (by the iterated expectations),

$$
\begin{aligned}
\mathrm{E}(\tilde{x} \tilde{q} \mid N D, Y=1) & =\mathrm{E}\left(\tilde{x} \tilde{q} \mid \tilde{q}<q_{\tilde{x}}\right)=\mathrm{E}\left[\mathrm{E}\left(\tilde{x} \tilde{q} \mid \tilde{q}<q_{\tilde{x}}, \tilde{x}\right) \mid \tilde{q}<q_{\tilde{x}}\right]=\mathrm{E}\left[\tilde{x} \mathrm{E}\left(q \mid \tilde{q}<q_{\tilde{x}}, \tilde{x}\right) \mid \tilde{q}<q_{\tilde{x}}\right] \\
& =E\left[\tilde{x}\left(\frac{\int_{0}^{q_{\tilde{x}}} q g(q) \mathrm{d} q}{G\left(q_{\tilde{x}}\right)}\right) \mid \tilde{q}<q_{\tilde{x}}\right] .
\end{aligned}
$$

To solve the above conditional expectation we first calculate the conditional cumulative distribution function $\Delta(\cdot)$. In particular,

$$
\Delta(x) \equiv \mathrm{P}\left(\tilde{x} \leqslant x \mid \tilde{q}<q_{\tilde{x}}\right)=\frac{\mathrm{P}\left(\tilde{x} \leqslant x, \tilde{q}<q_{\tilde{x}}\right)}{\mathrm{P}\left(\tilde{q}<q_{\tilde{x}}\right)}=\frac{\int_{0}^{x} f(z)\left(\int_{0}^{q_{z}} g(q) \mathrm{d} q\right) \mathrm{d} z}{\int_{0}^{\infty} f(z)\left(\int_{0}^{q_{z}} g(q) \mathrm{d} q\right) \mathrm{d} z} \quad \text { for } x \geqslant 0 .
$$

This implies that the density function of $\tilde{x}$, conditional on $\tilde{q}<q_{\tilde{x}}$, denoted by $\delta(\cdot)$, is given by

$$
\delta(x)=\Delta^{\prime}(x)=\frac{f(x) G\left(q_{x}\right)}{\int_{0}^{\infty} f(z) G\left(q_{z}\right) \mathrm{d} z}, \text { for } x \geqslant 0 .
$$

We can now continue to derive the conditional expectation $\mathrm{E}(\tilde{x} \tilde{q} \mid N D, Y=1)$ in (14) as

$$
\mathrm{E}\left[\tilde{x}\left(\frac{\int_{0}^{q_{\tilde{x}}} q g(q) \mathrm{d} q}{G\left(q_{\tilde{x}}\right)}\right) \mid \tilde{q}<q_{\tilde{x}}\right]=\frac{1}{\int_{0}^{\infty} f(z) G\left(q_{z}\right) \mathrm{d} z} \int_{0}^{\infty} x\left(\int_{0}^{q_{x}} q g(q) \mathrm{d} q\right) f(x) \mathrm{d} x .
$$

The expression for $\mathrm{P}(N D)$ as stated in the lemma follows after making the necessary substitutions.

Lemma A2. If for all $x \geqslant 0$, the function $Q_{x}:(0, \infty) \rightarrow[0,1]$ is continuous, then there exists $z^{*}>0$ such that $\Gamma\left(z^{*}\right)=0$, where $\Gamma:(0, \infty) \rightarrow R$ is given by,

$$
\begin{aligned}
& \Gamma(z) \equiv z-\psi(z)\left[(1-\lambda) \mathrm{E}(x) \mathrm{E}(q)+\lambda \int_{0}^{\infty} x f(x)\left(\int_{0}^{Q_{x}(z)} q g(q) \mathrm{d} q\right) \mathrm{d} x\right], \\
& \text { where } \psi(z)=\frac{1}{1-\lambda+\lambda \int_{0}^{\infty} G\left(Q_{x}(z)\right) f(x) \mathrm{d} x} .
\end{aligned}
$$

Proof of Lemma A2. We first note that for $z>0$,

$$
z-\frac{\mathrm{E}(x) \mathrm{E}(q)}{1-\lambda} \leqslant \Gamma(z) \leqslant z-(1-\lambda) \mathrm{E}(x) \mathrm{E}(q) .
$$

It can be shown with some algebra that the lower bound follows from $\psi(z) \leqslant \frac{1}{1-\lambda}$, and the upper bound follows from $\psi(z) \geqslant 1$. Thus, $\Gamma(z) \geqslant z-\frac{1}{1-\lambda} \mathrm{E}(x) \mathrm{E}(q)>0$ for $z \in\left(\frac{\mathrm{E}(x) \mathrm{E}(q)}{1-\lambda}, \infty\right)$, and $\Gamma(z) \leqslant z-(1-\lambda) \mathrm{E}(x) \mathrm{E}(q)<0$ for $z \in(0,(1-\lambda) \mathrm{E}(x) \mathrm{E}(q))$. Consider the two points: $z_{1} \in(0,(1-\lambda) \mathrm{E}(x) \mathrm{E}(q))$ and $z_{2} \in\left(\frac{\mathrm{E}(x) \mathrm{E}(q)}{1-\lambda}, \infty\right)$. If $\Gamma(z)$ is a continuous function over the bounded interval $\left[z_{1}, z_{2}\right]$, then by the Intermediate Value Theorem, there exists a $z^{*} \in\left(z_{1}, z_{2}\right)$, such that $\Gamma\left(z^{*}\right)=0$. Continuity of $\Gamma(\cdot)$ follows from the continuity of the cumulative distribution function $G(\cdot)$, and the continuity of $Q_{x}(\cdot)$ (the continuity of $Q_{x}(\cdot)$ for all $x \geqslant 0$ is stated as a condition in the lemma).

Proof of Proposition 1 (Disclosure equilibrium). We start the proof by showing that Eqs. (5)-(7) hold in equilibrium. It follows from Lemma 1 and (4) that for all $x$, the equilibrium disclosure cutoff $q_{x}$ is defined by (5) - for a given price following disclosure of $x, \mathrm{P}(x)$, and non-disclosure price, $\mathrm{P}(N D)$. The market price following a disclosure of $x\left(q_{x} \in[0,1)\right)$ equals $\mathrm{P}(x)$, as in (7) (see Lemma A1). For $x \in N D$, disclosure of $x$ does 
not occur in equilibrium, and the price $\mathrm{P}(x)$ represents an out-of-equilibrium price. ${ }^{20}$ Finally, the expected value of the terminal payoff $v$, conditional on non-disclosure (given cutoffs $\left\{q_{x}\right\}_{x \geqslant 0}$ ) equals $\mathrm{P}(N D)$, as in (6) (see Lemma A1).

Next we show existence of an equilibrium. For any $x$, define the function $Q_{x}:(0, \infty) \rightarrow[0,1]$, representing the disclosure threshold in terms of $q$ for a given non-disclosure price $\mathrm{P}(N D)>0$, as follows (in this definition, according to (7), we substitute $\mathrm{P}(x)=x$ for $x \in N D, \mathrm{P}(x)=x \mathrm{E}(q)$ for $x \in D$, and $\mathrm{P}(x)=x \mathrm{E}\left(q \mid q \geqslant Q_{x}(\mathrm{P}(N D))\right)$ for $x \in P D$, into (5)):

$$
Q_{x}(\mathrm{P}(N D))= \begin{cases}1 & \text { if } \mathrm{P}(N D) \geqslant x, \\ 0 & \text { if } \mathrm{P}(N D) \leqslant\left(1-\gamma_{x}\right) x \mathrm{E}(q), \\ \frac{\mathrm{P}(N D)-\left(1-\gamma_{x}\right) x \mathrm{E}\left(q \mid q \geqslant Q_{x}(\mathrm{P}(N D))\right)}{\gamma_{x} x} & \text { if otherwise. }\end{cases}
$$

We begin by establishing that such a function $Q_{x}(\mathrm{P}(N D))$ exists, is unique, and is continuous in $\mathrm{P}(N D)$. If $\mathrm{P}(N D) \geqslant x$, then $Q_{x}(\mathrm{P}(N D))=1$ is a solution. If $\mathrm{P}(N D) \leqslant\left(1-\gamma_{x}\right) x \mathrm{E}(q)$, then $Q_{x}(\mathrm{P}(N D))=0$ is a solution. These solutions are unique, as the set $\left\{\mathrm{P}(N D)>0: \mathrm{P}(N D) \geqslant x, \mathrm{P}(N D) \leqslant\left(1-\gamma_{x}\right) x \mathrm{E}(q)\right\}$ is empty.

Next, we consider the interval $\mathrm{P}(N D) \in\left(\left(1-\gamma_{x}\right) x \mathrm{E}(q), x\right)$. There exists a unique solution $Q_{x}(\mathrm{P}(N D))=$ $z^{*} \in(0,1)$ to the equation $H\left(z^{*}\right)=0$ where,

$$
H(z)= \begin{cases}\gamma_{x} x z+\left(1-\gamma_{x}\right) x \mathrm{E}(q \mid q \geqslant z)-\mathrm{P}(N D) & \text { for } z \in[0,1), \\ x-\mathrm{P}(N D) & \text { for } z=1 .\end{cases}
$$

Existence follows from the Intermediate Value Theorem, as $H(0)=\left(1-\gamma_{x}\right) x \mathrm{E}(q)-\mathrm{P}(N D)<0, H(1)=$ $x-\mathrm{P}(N D)>0$, and $H(z)$ is a continuous function in $z$ over the bounded interval $[0,1]$ (continuity follows from $\left.\lim _{z \rightarrow 1^{-}} \mathrm{E}(q \mid q \geqslant z)=1\right)$. Uniqueness of the solution $Q_{x}(\mathrm{P}(N D))=z^{*}$ follows from the monotonicity of $H(z)$ in $z \in(0,1)^{21}$ :

$$
H^{\prime}(z)=\gamma_{x} x+\left(1-\gamma_{x}\right) x \frac{\partial}{\partial z} \mathrm{E}(q \mid q \geqslant z)>0 \quad \text { for } z \in(0,1) .
$$

It remains to be shown that the solution $Q_{x}(\mathrm{P}(N D))$ is continuous in $\mathrm{P}(N D)$. To establish continuity, we consider three regions: $\mathrm{P}(N D)>x, \mathrm{P}(N D)<\left(1-\gamma_{x}\right) x \mathrm{E}(q)$, and $\mathrm{P}(N D) \in\left(\left(1-\gamma_{x}\right) x \mathrm{E}(q), x\right)$ and two points: $\mathrm{P}(N D)=x$, and $\mathrm{P}(N D)=\left(1-\gamma_{x}\right) x \mathrm{E}(q)$.

First, when $Q_{x}(\mathrm{P}(N D))$ is constant, it is also continuous (i.e., for $\mathrm{P}(N D)>x$ and $\left.\mathrm{P}(N D)<\left(1-\gamma_{x}\right) x \mathrm{E}(q)\right)$ ). For $\mathrm{P}(N D) \in\left(\left(1-\gamma_{x}\right) x \mathrm{E}(q), x\right)$ we use the Inverse Function Theorem. Let $\mathrm{P}_{N D}(z):(0,1) \rightarrow\left(\left(1-\gamma_{x}\right) x \mathrm{E}(q), x\right)$ where $\mathbf{P}_{N D}(z) \equiv \gamma_{x} x z+\left(1-\gamma_{x}\right) x \mathrm{E}(q \mid q \geqslant z)$. As $\mathbf{P}_{N D}(z)$ is continuously differentiable and strictly monotonic over the interval $z \in(0,1)$ it follows from the Inverse Function Theorem that its inverse $Q_{x}(\cdot)=\mathrm{P}_{N D}^{-1}(\cdot)$ is a continuously differentiable, strictly monotonic function from $\left(\left(1-\gamma_{x}\right) x \mathrm{E}(q), x\right)$ to $(0,1)$.

To establish continuity of $Q_{x}(\mathrm{P}(N D))$ at the points $\mathrm{P}(N D)=x$ and $\mathrm{P}(N D)=\left(1-\gamma_{x}\right) x \mathrm{E}(q)$, we compare the limits from above to the limits from below of the function $Q_{x}(\mathrm{P}(N D))$ at these points. The function is continuous, because $Q_{x}(\mathrm{P}(N D))$ approaches 1 as $\mathrm{P}(N D)$ approaches $x$, and $Q_{x}(\mathrm{P}(N D))$ approaches 0 as $\mathrm{P}(N D)$

\footnotetext{
${ }^{20}$ Any beliefs regarding $q$ (following a disclosure of $x \in N D$ ) would imply $\mathrm{P}(x) \leqslant x$. For robustness, we consider an equilibrium in which the price $\mathrm{P}(x)$ is most supportive of a deviation. The price that rewards deviation (in this case disclosure of $x)$ the most is $\mathrm{P}(x)=x$. It is worth noting that the equilibrium under these beliefs holds under any alternative beliefs, as it only depends on the property $\mathrm{P}(x) \leqslant x$.

${ }^{21}$ Note that for $y \in[0,1)$ the derivative $\frac{\partial \mathrm{E}(q \mid q \geqslant y)}{\partial y}>0$, as

$$
\begin{aligned}
& \frac{\partial \mathrm{E}(q \mid q \geqslant y)}{\partial y}=\frac{\partial}{\partial y}\left[\frac{1}{(1-G(y))} \int_{y}^{1} q g(q) \mathrm{d} q\right] \\
& \quad=\frac{1}{(1-G(y))^{2}}\left[-g(y) y(1-G(y))+g(y) \int_{y}^{1} q g(q) \mathrm{d} q\right] \\
& \quad=\frac{g(y)}{(1-G(y))^{2}}\left[\int_{y}^{1}(q-y) g(q) \mathrm{d} q\right]>0 .
\end{aligned}
$$
}

Moreover, the interval $\mathrm{P}(N D) \in\left(\left(1-\gamma_{x}\right) x \mathrm{E}(q), x\right)$ is non-empty only for $x>0$, and thus the derivative $H^{\prime}(z)$ is strictly positive. 
approaches $\left(\left(1-\gamma_{x}\right) x \mathrm{E}(q)\right) .^{22}$ Thus, we have established that the solution $Q_{x}(\mathrm{P}(N D))$ exists, is unique, and is continuous in $\mathrm{P}(N D)$.

Finally, in order to show that an equilibrium exists (i.e., that Eqs. (5)-(7) have a solution), it suffices to show that for $q_{x}=Q_{x}(\mathrm{P}(N D))$ there in fact exists a $\mathrm{P}(N D)$ that solves the equilibrium condition (6). That is, there exists a solution $z^{*}>0$ to $\Gamma\left(z^{*}\right)=0$, where $\Gamma:(0, \infty) \rightarrow R$, is given by

$$
\begin{aligned}
& \Gamma(z) \equiv z-\psi(z)\left[(1-\lambda) \mathrm{E}(x) \mathrm{E}(q)+\lambda \int_{0}^{\infty} x f(x)\left(\int_{0}^{Q_{x}(z)} q g(q) \mathrm{d} q\right) \mathrm{d} x\right] \\
& \text { where } \quad \psi(z)=\frac{1}{1-\lambda+\lambda \int_{0}^{\infty} G\left(Q_{x}(z)\right) f(x) \mathrm{d} x} .
\end{aligned}
$$

The existence of such $z^{*}$ follows directly from Lemma A2, as $Q_{x}(z)$ is continuous. An equilibrium, therefore, is given by a non-disclosure price $\mathrm{P}(N D)=\left(z^{*}>0\right.$ where $\Gamma\left(z^{*}\right)=0$, disclosure threshold levels $\left\{q_{x}\right\}_{x \geqslant 0}$ where $q_{x}=Q_{x}\left(z^{*}\right)$, and prices $\mathrm{P}(x)=x \mathrm{E}\left(q \mid q \geqslant Q_{x}\left(z^{*}\right)\right)$ following disclosure of $x \in D \cup P D$.

Proof of Corollary 1. First, the non-disclosure set $N D$ contains the interval [0, $\mathrm{P}(N D)]$, and is not an empty set. To see this, consider $\hat{x} \in[0, \mathrm{P}(N D)]$. It follows from (7) that

$$
\gamma_{\hat{x}} \hat{x}+\left(1-\gamma_{\hat{x}}\right) P(\hat{x}) \leqslant \hat{x} \leqslant \mathrm{P}(N D),
$$

and therefore it follows from (5) that $q_{\hat{x}}=1$ (recall from (6) that $\mathrm{P}(N D)>0$ ).

Second, the full disclosure set, $D$, contains the interval $\left[\frac{\mathrm{P}(N D)}{(1-\bar{\gamma}) \mathrm{E}(q)}, \infty\right)$, and is not an empty set. To see this, consider $\hat{x} \in\left[\frac{\mathrm{P}(N D)}{(1-\bar{\gamma}) \mathrm{E}(q)}, \infty\right)$, and note that $\hat{x} \geqslant \frac{\mathrm{P}(N D)}{(1-\bar{\gamma}) \mathrm{E}(q)} \geqslant \frac{\mathrm{P}(N D)}{\left(1-\gamma_{\hat{x}}\right) \mathrm{E}(q)}\left(\right.$ recall that for all $x \geqslant 0, \gamma_{x} \in[0, \bar{\gamma})$ where $\left.\bar{\gamma}<1\right)$. Therefore, it follows from (5) that $q_{\hat{x}}=0$.

Finally, the partial disclosure set, $P D$, contains the interval $\left(\mathrm{P}(N D), \frac{\mathrm{P}(N D)}{\mathrm{E}(q)}\right)$, and is not an empty set. To see this, first note that for $x \in D$, we have $q_{x}=0$, and it follows (from (5) and (7)) that

$$
\left(1-\gamma_{x}\right) \mathrm{P}(x) \geqslant \mathrm{P}(N D)
$$

and

$$
\mathrm{P}(x)=x \mathrm{E}(q) .
$$

Thus, if $x \in D$, then it must be that $x \geqslant \frac{\mathrm{P}(N D)}{\left(1-\gamma_{x}\right) \mathrm{E}(q)}$. Second, note that for $x \in N D$, we have $q_{x}=1$, and it follows (from (5) and (7)) that

$$
\gamma_{x} x+\left(1-\gamma_{x}\right) \mathrm{P}(x) \leqslant \mathrm{P}(N D)
$$

and

$$
\mathrm{P}(x)=x .
$$

Thus, if $x \in N D$, then it must be that $x \leqslant \mathrm{P}(N D)$. Therefore, for any $x^{0} \in\left(\mathrm{P}(N D), \frac{\mathrm{P}(N D)}{\mathrm{E}(q)}\right)$ it must be that $x^{0} \notin$ $D \cup N D$ (as $\left.\frac{\mathrm{P}(N D)}{\mathrm{E}(q)} \leqslant \frac{\mathrm{P}(N D)}{\left(1-\gamma_{x^{0}}\right) \mathrm{E}(q)}\right)$, i.e., $x^{0} \in P D$, and $P D$ is not an empty set.

Proof of Proposition 2 (Disclosure equilibrium with a strategic analyst). As in the proof of Proposition 1, Eqs. (5)-(7) must hold in equilibrium. Moreover, for any $x$, the analyst's best response to the manager's disclosure threshold $q_{x}$ is given by the first order condition (11). Due to the strict convexity of $c$, the inverse function $c^{\prime-1}$ is well defined, and we can implicitly define a unique analysts' response, $\gamma_{x}=\Upsilon\left(q_{x}\right)$, to a disclosure of $x \in P D \cup D$,

$$
r\left(q_{x}\right)=c^{-1}\left(\frac{\mathrm{E}\left(q^{2} \mid q \geqslant q_{x}\right)}{\mathrm{E}^{2}\left(q \mid q \geqslant q_{x}\right)}-1\right)
$$

\footnotetext{
${ }^{22}$ Note that $\gamma_{x} x z+\left(1-\gamma_{x}\right) x \mathrm{E}(q \mid q \geqslant z)$ is strictly increasing in $z$, and as a result $z^{*}$ is increasing in $\mathrm{P}(N D)$, for $\mathrm{P}(N D) \in\left(\left(1-\gamma_{x}\right) x \mathrm{E}(q), x\right)$. Due to the monotonicity of $z^{*}$ in $\mathrm{P}(N D)$, as $\mathrm{P}(N D) \rightarrow x, z^{*} \rightarrow 1$, and as $\mathrm{P}(N D) \rightarrow\left(1-\gamma_{x}\right) x \mathrm{E}(q), z^{*} \rightarrow 0$.
} 
Define also $Y(1)=0$, as the level of scrutiny following no disclosure. For $x \in N D$, disclosure of $x$ does not occur in equilibrium, and the level of analyst scrutiny $\gamma_{x}=\Upsilon(1)$ represents an out-of-equilibrium level of scrutiny. ${ }^{23}$

Before we continue, it is useful to establish a few properties of the function $Y(z)$. First, Note that $Y(z)$ is continuous over the bounded interval [0,1], as $\lim _{z \rightarrow 1^{-}}\left(\frac{\mathrm{E}\left(q^{2} \mid q \geqslant z\right)}{\mathrm{E}^{2}(q \mid q \geqslant z)}-1\right)=0$. Second, it follows from Assumption A1 and Lemma 2 (i.e., $\frac{\partial}{\partial q_{x}}\left[\frac{\mathrm{E}\left(q^{2} \mid q \geqslant q_{x}\right)}{\mathrm{E}^{2}\left(q \mid q \geqslant q_{x}\right)}\right]<0$ ), and the strict convexity of $c(\gamma)$, that $\Upsilon^{*}(z)<0$. In particular, according to the Implicit Function Theorem,

$$
\frac{\mathrm{d} \Upsilon\left(q_{x}\right)}{\mathrm{d} q_{x}}=\frac{\frac{\partial}{\partial q_{x}}\left[\frac{\mathrm{E}\left(q^{2} \mid q \geqslant q_{x}\right)}{\mathrm{E}^{2}\left(q \mid q \geqslant q_{x}\right)}\right]}{c^{\prime \prime}\left(\Upsilon\left(q_{x}\right)\right)}<0 \quad\left(q_{x} \in[0,1)\right) .
$$

We do not present the rest of this proof has for the sake of brevity-we can proceed exactly as in the proof of Proposition 1 by replacing the exogenously assumed $\gamma_{x}$ in that proof by the analyst's response function $Y\left(q_{x}\right)$ as characterized above.

Proof of Corollary 2. The result follows from $\frac{\mathrm{d} r\left(q_{x}\right)}{\mathrm{d} q_{x}}<0$ as derived in the proof of Proposition 2 (see (16) for definition of the function 1 ). Thus, for any $x, y \geqslant 0$ we have $q_{x}>q_{y} \Leftrightarrow \gamma_{x}<\gamma_{y}$.

Proof of Corollary 3. First, we show that $x \leqslant \mathrm{P}(N D) \Longrightarrow q_{x}=1$. It follows from $x \leqslant \mathrm{P}(N D)$ and $\mathrm{P}(x) \leqslant x$ that $\gamma_{x} x+\left(1-\gamma_{x}\right) \mathrm{P}(x) \leqslant x \leqslant \mathrm{P}(N D)$. Thus, from (5), $q_{x}=1$. Next, we show that $q_{x}=1 \Longrightarrow x \leqslant \mathrm{P}(N D)$. Notice that when $q_{x}=1$, it follows from (5) that $\mathrm{P}(N D) \geqslant \gamma_{x} x+\left(1-\gamma_{x}\right) \mathrm{P}(x)$. From (7) and $q_{x}=1$, it also follows that $\mathrm{P}(x)=x$. Thus, $\mathrm{P}(N D) \geqslant \gamma_{x} x+\left(1-\gamma_{x}\right) \mathrm{P}(x)=x$. We can conclude that $x \leqslant \mathrm{P}(N D) \Leftrightarrow q_{x}=1$, or equivalently $N D=[0, \mathrm{P}(N D)]$.

Proof of Corollary 4. Before we begin the proof, recall the following properties: for all $x \geqslant 0, \mathrm{P}(x) \geqslant x \mathrm{E}(q)$ (from (7)), and $\gamma_{x}=\Upsilon\left(q_{x}\right) \leqslant \Upsilon(0)$ (from Corollary 2). Let $\bar{x}=\frac{\mathrm{P}(N D)}{\mathrm{E}(q)(1-r(0))}(\bar{x}<\infty$ as $\Upsilon(0) \in[0, \bar{\gamma})$ ). First we show that $x \geqslant \bar{x} \Longrightarrow q_{x}=0$. For all $x \geqslant \bar{x}$, we have $\mathrm{P}(x)\left(1-\gamma_{x}\right) \geqslant x \mathrm{E}(q)\left(1-\gamma_{x}\right) \geqslant \bar{x} \mathrm{E}(q)(1-\gamma(0))=\mathrm{P}(N D)$. Thus, it follows from (5) that $q_{x}=0$.

Next, we show that $q_{x}=0 \Longrightarrow x \geqslant \bar{x}$. It follows from (16) (in the proof of Proposition 2) that $\gamma_{x}=Y(0)$, and it follows from (7) that $\mathrm{P}(x)=x \mathrm{E}(q)$. Finally, it follows from (5) that $\mathrm{P}(N D) \leqslant \mathrm{P}(x)\left(1-\gamma_{x}\right)=$ $x \mathrm{E}(q)(1-Y(0))$ which is satisfied if and only if $x \geqslant \frac{\mathrm{P}(N D)}{\mathrm{E}(q)(1-\gamma(0))}=\bar{x}$. Thus, we can conclude that $x \geqslant \bar{x} \Leftrightarrow q_{x}=0$, or equivalently $D=\left[\frac{\mathrm{P}(N D)}{\mathrm{E}(q)(1-r(0))}, \infty\right)$.

Proof of Corollary 5. As $N D=[0, \mathrm{P}(N D)]$ (from Corollary 3), $D=\left[\frac{\mathrm{P}(N D)}{\mathrm{E}(q)(1-r(0))}, \infty\right)$ (from Corollary 4), and $R^{+}=N D \cup P D \cup D$, it follows that $P D=\left(\mathrm{P}(N D), \frac{\mathrm{P}(N D)}{\mathrm{E}(q)(1-r(0))}\right)$. Moreover, the set $P D$ is not an empty set as $\mathrm{P}(N D)>0$, and $\mathrm{E}(q)(1-Y(0)) \in(0,1)$.

Proof of Proposition 3 (Disclosure informativeness). Recall from Corollary 3 that $N D=[0, \mathrm{P}(N D)]$ (i.e., $q_{x}=$ 1 in this region) and recall from Corollary 4 that $D=\left[\frac{\mathrm{P}(N D)}{\mathrm{E}(q)(1-r(0))}, \infty\right)$ (i.e., $q_{x}=0$ in this region). Next, define the function $\digamma:\left(\mathrm{P}(N D), \frac{\mathrm{P}(N D)}{\mathrm{E}(q)(1-r(0))}\right) \times(0,1) \rightarrow R$ given by

$$
\digamma(x, z)=\mathrm{P}(N D)-\Upsilon(z) x z-(1-\Upsilon(z)) x \mathrm{E}(q \mid q \geqslant z),
$$

where $Y(z)$ is as defined in (16). In equilibrium, from (5), $\digamma\left(x, q_{x}\right)=0$ for $x \in P D$. We appeal to the Implicit Function Theorem to establish that $\frac{\mathrm{d} q_{x}}{\mathrm{~d} x}<0$ for $x \in P D$. In particular, $\frac{\mathrm{d} q_{x}}{\mathrm{~d} x}=-\frac{\partial \digamma\left(x, q_{x}\right)}{\partial x} / \frac{\partial \digamma\left(x, q_{x}\right)}{\partial q_{x}}<0$. The result follows from (recall that $Y^{\prime}\left(q_{x}\right)<0$ )

$$
\frac{\partial \digamma\left(x, q_{x}\right)}{\partial x}=-\eta\left(q_{x}\right) q_{x}-\left(1-\eta\left(q_{x}\right)\right) \mathrm{E}\left(q \mid q \geqslant q_{x}\right)<0
$$

\footnotetext{
${ }^{23}$ Any beliefs regarding $q$ (following a disclosure of $x \in N D$ ) would imply $\gamma_{x} \in[0, \bar{\gamma})$. In line with our treatment of $\mathrm{P}(x)$ for $x \in N D$, for robustness, we consider an equilibrium that is most supportive of a deviation. The level of analyst scrutiny that rewards deviation, i.e., disclosure of $x \in N D$ for some $q \in[0,1]$, the most is $\gamma_{x}=0$ (recall that for $\left.x \in N D, \mathrm{P}(x)=x \geqslant v\right)$. It is worth noting that the equilibrium under these beliefs holds under any alternative beliefs, as it depends on the property $\gamma_{x} x+\left(1-\gamma_{x}\right) \mathrm{P}(x) \leqslant x$ that holds for any $\gamma_{x} \in[0,1]$, and $x \in N D$.
} 
and

$$
\frac{\partial \digamma\left(x, q_{x}\right)}{\partial q_{x}}=-x \Upsilon\left(q_{x}\right)-x\left(1-\Upsilon\left(q_{x}\right)\right) \frac{\partial \mathrm{E}\left(q \mid q \geqslant q_{x}\right)}{\partial q_{x}}+x Y^{*}\left(q_{x}\right)\left(\mathrm{E}\left(q \mid q \geqslant q_{x}\right)-q_{x}\right)<0 .
$$

Thus, we can conclude that for any $y>x \geqslant 0$ we have $q_{x} \geqslant q_{y}$, and for any $y>x>z \geqslant 0$ where $x \in P D$ we have $q_{z}>q_{x}>q_{y}$.

Proof of Proposition 4 (Analyst scrutiny). It follows from Corollary 2 that for any $x, y \geqslant 0$ we have $q_{x}>q_{y} \Leftrightarrow \gamma_{x}=r\left(q_{x}\right)<\Upsilon\left(q_{y}\right)=\gamma_{y}$. It follows from Proposition 3 that for any $y>x \geqslant 0$ we have $q_{x} \geqslant q_{y}$ which then leads to $\gamma_{x}=\Upsilon\left(q_{x}\right) \leqslant Y\left(q_{y}\right)=\gamma_{y}$, thus the level of analyst scrutiny is weakly increasing in the disclosure $x$. Moreover, if $y>x>z \geqslant 0$ for some $x \in P D$ then it follows from Proposition 3 that $q_{z}>q_{x}>q_{y}$ which then leads to $\gamma_{y}=\Upsilon\left(q_{y}\right)>\gamma_{x}=\Upsilon\left(q_{x}\right)>\Upsilon\left(q_{z}\right)=\gamma_{z}$.

Proof of Proposition 5 (Stock price response to disclosure). Consider first $x \in D=\left[\frac{\mathrm{P}(N D)}{\mathrm{E}(q)(1-\gamma(0))}, \infty\right)$, for which the price following disclosure of $x$ is $\mathrm{P}(x)=x \mathrm{E}(q)$, i.e., $\mathrm{P}(x)$ is strictly increasing in $x$. Consider next $x \in P D=\left(\mathrm{P}(N D), \frac{\mathrm{P}(N D)}{\mathrm{E}(q)(1-r(0))}\right)$, and let $H:\left(\mathrm{P}(N D), \frac{\mathrm{P}(N D)}{\mathrm{E}(q)(1-r(0))}\right) \rightarrow R$, be given by,

$$
H(x)=\Upsilon\left(q_{x}\right) x q_{x}+\left(1-\Upsilon\left(q_{x}\right)\right) x \mathrm{E}\left(q \mid q \geqslant q_{x}\right) .
$$

It follows from Proposition 2 that $H(x)=\mathrm{P}(N D)$, and $\frac{\partial H(x)}{\partial x}=0$, for all $x \in P D$, where

$$
\frac{\partial H(x)}{\partial x}=x \frac{\partial \Upsilon\left(q_{x}\right)}{\partial q_{x}} \frac{\partial q_{x}}{\partial x}\left[q_{x}-\mathrm{E}\left(q \mid q \geqslant q_{x}\right)\right]+\Upsilon\left(q_{x}\right)\left[\frac{\mathrm{d}\left(x q_{x}\right)}{\mathrm{d} x}-\frac{\mathrm{d}\left(x \mathrm{E}\left(q \mid q \geqslant q_{x}\right)\right)}{\mathrm{d} x}\right]+\frac{\mathrm{d}\left(x \mathrm{E}\left(q \mid q \geqslant q_{x}\right)\right)}{\mathrm{d} x} .
$$

To show that $\frac{\partial \mathrm{P}(x)}{\partial x}=\frac{\mathrm{d}\left(x \mathrm{E}\left(q \mid q \geqslant q_{x}\right)\right)}{\mathrm{d} x}>0$, note that by Assumption A1 $\left(\frac{\partial \mathrm{E}\left(q \mid q \geqslant q_{x}\right)}{\partial q_{x}} \in(0,1)\right)$, Proposition $3\left(\frac{\partial q_{x}}{\partial x}<0\right.$ for all $x \in P D)$, and $\mathrm{E}\left(q \mid q \geqslant q_{x}\right) \geqslant q_{x}$, it follows that,

$$
\frac{\mathrm{d}\left(x q_{x}\right)}{\mathrm{d} x}=q_{x}+x \frac{\partial q_{x}}{\partial x} \leqslant \mathrm{E}\left(q \mid q \geqslant q_{x}\right)+x \frac{\partial \mathrm{E}\left(q \mid q \geqslant q_{x}\right)}{\partial q_{x}} \frac{\partial q_{x}}{\partial x}=\frac{\mathrm{d}\left(x \mathrm{E}\left(q \mid q \geqslant q_{x}\right)\right)}{\mathrm{d} x} .
$$

Reorganizing $\frac{\partial H(x)}{\partial x}=0$ implies that,

$$
\frac{\mathrm{d}\left(x \mathrm{E}\left(q \mid q \geqslant q_{x}\right)\right)}{\mathrm{d} x}=-x \frac{\partial \Upsilon\left(q_{x}\right)}{\partial q_{x}} \frac{\partial q_{x}}{\partial x}\left[q_{x}-\mathrm{E}\left(q \mid q \geqslant q_{x}\right)\right]+\Upsilon\left(q_{x}\right)\left[\frac{\mathrm{d}\left(x \mathrm{E}\left(q \mid q \geqslant q_{x}\right)\right)}{\mathrm{d} x}-\frac{\mathrm{d}\left(x q_{x}\right)}{\mathrm{d} x}\right]>0 .
$$

The strict inequality follows from $\frac{\partial r\left(q_{x}\right)}{\partial q_{x}}<0, \frac{\partial q_{x}}{\partial x}<0,\left[q_{x}-\mathrm{E}\left(q \mid q \geqslant q_{x}\right)\right]<0$, and $\frac{\mathrm{d}\left(x q_{x}\right)}{\mathrm{d} x} \leqslant \frac{\mathrm{d}\left(x \mathrm{E}\left(q \mid q \geqslant q_{x}\right)\right)}{\mathrm{d} x}$. Thus, we conclude that $\frac{\mathrm{dP}(x)}{\mathrm{d} x}=\frac{\mathrm{d}\left(x \mathrm{E}\left(q \mid q \geqslant q_{x}\right)\right)}{\mathrm{d} x}>0$, for $x \in P D \cup D$.

Proposition 6 (Disclosure equilibrium under an accounting system with precision $\theta$ ). For an accounting system characterized by $\theta \in[0, \bar{\theta})$, there exists a voluntary disclosure equilibrium consisting of a set of thresholds $\left\{q_{x}\right\}_{x \geqslant 0}$, a set of prices $\mathrm{P}(N D)$ and $\{\mathrm{P}(x)\}_{x \geqslant 0}$, and the degree of analyst scrutiny $\left\{\gamma_{x}\right\}_{x \geqslant 0}$ such that:

I. If the manager learns $\langle x, q\rangle$, then he discloses $x$ only if $q \geqslant q_{x}$, as in (12).

II. The market rationally sets prices $\mathrm{P}(N D)$ as in (6) and $\mathrm{P}(x)$ as in (7).

III. Upon observing $x$ when disclosed, the analyst chooses the degree of scrutiny $\gamma_{x}$ as in (11).

Proof of Proposition 6 and Corollary 6. Incorporating an accounting system does not directly affect market prices following disclosure and non-disclosure - i.e., equilibrium conditions (6) and (7). The analyst's best response function (see details in the body of the text) does not depend on $\theta$ either-i.e., equilibrium condition (11). The manager's best response disclosure strategy, however, depends on $\theta$. In particular, the probability of discovery by the analyst is now $\gamma_{x}+\theta$-i.e., equilibrium condition (12). The proof of Proposition 7 is qualitatively the same as that of Proposition 2, and the proofs of Corollaries 2-5 and Propositions 3-4 can be repeated to incorporate an accounting system without any qualitative difference. For completeness, however, we redefine the upper limit of the partial disclosure set $P D$ which depends on $\theta$, as $\bar{x}=\frac{\mathrm{P}(N D)}{\mathrm{E}(q)(1-r(0)-\theta)}$. 
Lemma 3 (Disclosure equilibrium under an accounting system with precision $\theta(q)$ ). For an accounting system characterized by $\theta:[0,1] \rightarrow[0, \bar{\theta})$, there exists a voluntary disclosure equilibrium consisting of a set of thresholds $\left\{q_{x}\right\}_{x \geqslant 0}$, a set of prices $\mathrm{P}(N D)$ and $\{\mathrm{P}(x)\}_{x \geqslant 0}$, and the degree of analyst scrutiny $\left\{\gamma_{x}\right\}_{x \geqslant 0}$ such that:

I. If the manager learns $\langle x, q\rangle$, then he discloses $x$ only if $q \geqslant q_{x}$, where

$$
q_{x}= \begin{cases}1, & \text { if } \mathrm{P}(N D)-\left(1-\gamma_{x}-\theta(1)\right) \mathrm{P}(x) \geqslant\left(\gamma_{x}+\theta(1)\right) x, \\ 0, & \text { if } \mathrm{P}(N D)-\left(1-\gamma_{x}-\theta(0)\right) \mathrm{P}(x) \leqslant 0, \\ \frac{\mathrm{P}(N D)-\left(1-\gamma_{x}-\theta\left(q_{x}\right)\right) \mathrm{P}(x)}{\left(\gamma_{x}+\theta\left(q_{x}\right)\right) x} & \text { if otherwise. }\end{cases}
$$

II. The market rationally sets prices $\mathrm{P}(N D)$ as in (6) and $\mathrm{P}(x)$ as in (7).

III. Upon observing $x$ when disclosed, the analyst chooses the degree of scrutiny $\gamma_{x}$ as in (11).

Lemma 4. For $\theta^{0}>\frac{3}{2}|b|$, and $c>\underline{c}=\frac{1}{3}\left(1-\theta^{0}-\frac{1}{2}|b|\right)^{-1}$, there exists a unique equilibrium with the following disclosure thresholds ${ }^{24}$ :

1. The manager never discloses when $x=0\left(q_{0}=1\right)$.

2. The manager will always disclose when $x=1\left(q_{1}=0\right)$ if $c \geqslant \bar{c}$, where $\bar{c}=\frac{1}{3}\left(1-\theta^{0}+\frac{b}{2}-p \frac{1-\lambda}{1-\lambda+\lambda(1-p)}\right)^{-1}$.

3. The manager will disclose whenever $q \geqslant q_{1}$ with $q_{1} \in\left(0, \frac{1}{2}\right)$ if $c \in(\underline{c}, \bar{c})$. Moreover, $\underline{c}<\bar{c}$ if $b<p \frac{1-\lambda}{1-\lambda+\lambda(1-p)}$.

\section{References}

Bartov, E., Givoly, D., Hayn, C., 2002. The rewards to meeting or beating earnings expectations. Journal of Accounting and Economics 33, 173-204.

Basu, S., 1997. The conservatism principle and the asymmetric timeliness of earnings. Journal of Accounting and Economics $24,3-37$. Bushee, B.J., Miller, G.S., 2006. Investor relations, firm visibility, and investor following. Working Paper, University of Pennsylvania.

Chen, Q., Hemmer, T., Zhang, Y., 2007. On the relation between conservatism in accounting standards and incentives for earnings management. Journal of Accounting Research 45, 541-565.

Dutta, S., Truman, B., 2002. The interpretation of information and corporate disclosure strategies. Review of Accounting Studies 7 , 75-96.

Dye, R., 1985. Disclosure of nonproprietary information. Journal of Accounting Research 23, 123-145.

Dye, R., 1986. Proprietary and nonproprietary disclosures. Journal of Business 59, 331-336.

Dye, R., 1988. Earnings management in overlapping generations model. Journal of Accounting Research 26, $195-235$.

Dye, R., 1990. Mandatory versus voluntary disclosures: the cases of financial and real externalities. The Accounting Review 65, 1-24.

Dye, R., 2001. An evaluation of 'Essays on Disclosure' and the disclosure literature in accounting. Journal of Accounting and Economics $32,181-235$.

Dye, R., Sridhar, S., 1995. Industry-wide disclosure dynamics. Journal of Accounting Research 33, $157-174$.

Dye, R., Sridhar, S., 2002. Resource allocation effects of price reactions to disclosures. Contemporary Accounting Research 19, 385-410.

Fishman, M., Hagerty, K., 1989. Disclosure decisions by firms and the competition for price efficiency. The Journal of Finance 44, 633-646.

Gigler, F., Hemmer, T., 2001. Conservatism, optimal disclosure policy, and the timeliness of financial reports. The Accounting Review 76, 471-493.

Jung, W., Kwon, Y., 1988. Disclosure when market is unsure of information endowment of managers. Journal of Accounting Research 26, 146-153.

Kothari, S., Shu, S., Wysocki, P., 2005. Do managers withhold bad news? Working Paper, MIT.

Kwon, Y., 2005. Accounting conservatism and managerial incentives. Management Science 51, 1626-1632.

Kwon, Y., Newman, P., Suh, Y.S., 2001. The demand for accounting conservatism for management control. Review of Accounting Studies 6, 29-52.

Lopez, T., Rees, L., 2002. The effect of beating and missing analysts' forecasts on the information content of unexpected earnings. Journal of Accounting, Auditing and Finance 17, 155-184.

\footnotetext{
${ }^{24}$ The condition $\theta^{0}>\frac{3|b|}{2}$ guarantees Assumption A2 and also implies that $\theta^{0}>\frac{|b|}{2}$ which guarantees that the increase in the probability of discovery $\theta(q)$ afforded by the accounting system is strictly positive $(\theta(q)>0$ for all $q \in[0,1])$. The condition on the analyst's search cost, $c>\underline{c}=\frac{1}{3}\left(1-\theta^{0}-\frac{|b|}{2}\right)^{-1}$, guarantees that the equilibrium probability of discovery is strictly less than $1\left(i . e ., \gamma_{1}+\theta(z)<1\right.$ for all $\left.z \in[0,1]\right)$.
} 
McNichols, M., O’Brien, P., 1997. Self-selection and analyst coverage. Journal of Accounting Research 35, $167-199$.

Mikhail, M.B., Walther, B.R., Willis, R.H., 1999. Does forecast accuracy matter to security analysts? The Accounting Review 74, $185-200$.

Narayanan, M., 1985. Managerial incentives for short-term results. Journal of Finance 40, 1469-1484.

Raghu, V., 2004. Conservatism in accounting: good or bad? Working Paper, University of Chicago.

Stein, J., 1989. Efficient capital markets, inefficient firms: a model of myopic corporate behavior. Quarterly Journal of Economics 104, 655-669.

Verrecchia, R., 1983. Discretionary disclosure. Journal of Accounting and Economics 5, 179-194.

Verrecchia, R., 1990. Information quality and discretionary disclosure. Journal of accounting and Economics 12, 365-380.

Verrecchia, R., 2001. Essays on disclosure. Journal of Accounting and Economics 32, 97-180.

Watts, R., 2003a. Conservatism in accounting part I: explanations and implications. Accounting Horizons 17, $207-221$.

Watts, R., 2003b. Conservatism in accounting part II: evidence and research opportunities. Accounting Horizons 17, $287-301$. 